Originalveröffentlichung in: Grenzmann, Ludger ; Haye, Thomas ; Henkel, Nikolaus ; Kaufmann, Thomas (Hrsgg.): Wechselseitige Wahrnehmung der Religionen im Spätmittelalter und in der frühen Neuzeit, II. Kulturelle Konkretionen (Literatur, Mythographie, Wissenschaft und Kunst), Berlin; Boston: De Gruyter 2012, S. 209-245 (Abhandlungen der Akademie der Wissenschaften zu Göttingen, Neue Folge ; 4)

\title{
Muslime im Bild des Spätmittelalters: Unterschiedliche Blicke auf die 'Anderen'1
}

\author{
LIESELOTTE E. SAURMA-JELTSCH
}

\section{Einleitung: Der wechselnde Blick auf ein Bild}

Der Papstbesuch in Deutschland 2006, insbesondere die Regensburger Rede, brachte ein mittelalterliches Bild wieder ins Gespräch, das seit 2001 mehrfach Ziel ikonoklastischer Akte gewesen war, nämlich die Höllendarstellung von Giovanni da Modena. Dem 1415 gemalten Fresko in San Petronio in Bologna verschafften Gazetten, Internetseiten und in der Folge auch wissenschaftliche Analysen $^{2}$ einen Bekanntheitsgrad, den es nahezu 600 Jahre nicht besessen hatte. Die in den Medien vielfach reproduzierte Darstellung ${ }^{3}$ des an einen Fels gefesselten nackten Propheten Mohammed wurde von muslimischen Gruppierungen trotz ihrer lange zurückliegenden Entstehung als unmittelbare Kränkung empfunden, die es auszumerzen und zu zerstören galt. ${ }^{4}$ Literarisch liefert Dante die

1 Der Beitrag wurde überarbeitet im Zusammenhang des Projektes: „Images of Alterity in East and West", gefördert mit Mitteln des im Rahmen der Exzellenzinitiative des Bundes und der Länder eingerichteten Exzellenzclusters der Universität Heidelberg „Asia and Europe in a Global Context: Shifting Asymmetries in Cultural Flows." In veränderter Form ist er auf Englisch unter dem Titel erschienen: Saracens. Opponents to the Body of Christianity. The Medieval History Journal 13/1, (2010) S. 55-95. Weiterführende Gedanken finden sich in meinem Beitrag The Metamorphic Other and the Discourse of Alterity in Parisian Miniatures of the Fourteenth Century. In: Images of Otherness in Medieval and Early Modern Times: Exclusion, Inclusion and Assimilation. Hg. von ANJA EisenBeiß und LieselotTe E. SAURMA-JeltsCh, Berlin 2012, S. 37-56, Pl. 1.11.10. - Anja Eisenbeiß M. A. und Annette Hoffmann M. A. sei für die inspirierenden Gespräche gedankt.

2 JULIAN NAMÉ: 'Muslimische Identität' und das explosive Vermächtnis des Multikulturalismus. Novo 82 (Mai / Juni 2006), URL: <http://www.novo-magazin.de/82/novo 8210.htm> (6.2.2009).

3 Archive 'San Petronio', 7. April 2006: Terrorist Attacks Thwarted in Italy, URL: <http://neveryetmelted.com/categories/san-petronio/> (6.2.2009),

4 Schon vor den Drohungen und Attacken gegen San Petronio hatte Adel Smith, Präsident der Unione dei Musulmani d'Italia gefordert, das Wandbild des Jüngsten Gerichts zu zerstören, da es kränkender sei als Salman Rushdies «Satanische Verse», dazu BERND WEILER: Das alte Europa, seine neuen Bürger und der Streit um religiös-weltanschauliche Symbole im öffentlichen Raum. In: Demographie. Bewegungen einer Gesellschaft im Ruhestand. Multidisziplinäre Perspektiven zur Demographiefolgenforschung. Hg. von 
berühmteste Vorlage für diese Einbindung Mohammeds in die Szenerie des Jüngsten Gerichts. Im neunten Höllenkreis ${ }^{5}$ sieht er unter den Stiftern von Gezänk und Zwietracht (scisma) auch den Propheten. Freilich war die Motivation zu dieser Darstellung hochpolitisch, ist doch der Prophet an prominenter Stelleim Vordergrund des Wandbildes direkt über dem Höllenschlund - nicht als Einzelfigur zu verstehen, sondern bildet mit dem ihm gegenüber auf einem zweiten Felsplateau liegenden Gegenpapst Nikolaus V. (Pietro Rainalducci) ${ }^{6}$ ein zusammengehöriges Paar. In polemischer Weise wird der auf Betreiben Kaiser Ludwigs des Bayern gegen Johannes XXII. eingesetzte Papst, der sich nur kurz halten konnte (1328-1330), schon sehr bald mit einem Mönch Niccolò verschmolzen, der sich angeblich dem Islam zugewandt haben soll. ${ }^{7}$ Insbesondere im Kontext der Kirchenspaltung wird diese Gestalt geschmäht, für das Schisma verantwortlich gemacht ${ }^{8}$ und gemeinsam mit Mohammed zum Inbegriff jener Häretiker, die zu einer Spaltung des Christentums führen. ${ }^{9}$ Die literarische Schilderung Dantes, die sich dem Zustand der 'gespaltenen' Körper widmet, wird in der Wandmalerei nach der üblichen Ikonographie des Jüngsten Gerichts gestaltet, wenn Teufel sich mit ihren Krallen in die Gliedmaßen der Schismatiker schlagen und sie so als Sinnbild ihrer eigenen zerstörerischen Tätigkeit mit tiefen Wunden versehen. ${ }^{10}$ Was für den gläubigen Muslim blasphemisch erscheint, gibt in einer in der westlichen Kunst vertrauten Bildersprache die Vorstellungen des ausge-

Stephan A. Jansen, Birger P. Priddat und Nico SteHr. Wiesbaden 2005 (ZU / Schriften der Zeppelin University zwischen Wirtschaft, Kultur und Politik), S. 322-361, hier 341.

5 Inferno XXVIII, 30 f.: dicendo: Or vedi com'io mi dilacco! / Vedi come storpiato è Maometto!, und 34-36: E tutti li altri che tu vedi qui / seminator di scandalo e di scisma / fuor vivi, $e$ però son fessi cosi. Zitiert nach Dante Alighieri: Commedia. Inferno. Hg. von EMILIO PASQUINI und ANTONIO QUAGLIO. Mailand 1982 (I grandi libri Garzanti. Band 274).

6 ANSGAR FRENKEN: Nikolaus V. In: Biographisch-Bibliographisches Kirchenlexikon VI (1993), Sp. 871-872.

7 ILKa KLOTEN: Wandmalerei im großen Kirchenschisma. Die Cappella Bolognini in San Petronio zu Bologna. Heidelberg 1986, S. $73 \mathrm{ff}$.

8 Joseph POlzer: Aristotle, Mohammed and Nicholas V in Hell. The Art Bulletin 46 (1964) S. 457-469, bes. 458.

9 Marnie Anjenette Leist: The Virgin and Hell. An Anomalous Fifteenth-Century Italian Mural. Master Thesis University of Cincinnati 2005 (ProQuest Dissertations \& Theses Full Text, Publication Number AAT 1429379), bes. S. 111 ff. Hier auch zum besonderen Interesse der Auftraggeber und der Kommune von Bologna an der Demonstration der Zugehörigkeit zu Johannes XXII. und dem damit verbundenen Häresievorwurf gegen Nikolaus V.

10 Ähnlich in den um 1300 entstandenen Archivolten von Santa Maria in Toro bei Zamora; dazu Yves CHRIST: Das Jüngste Gericht. Regensburg 2001 (Die Welt des Mittelalters), Abb. 161, Detail, S. 157, und im Sinne Dantes vor allem in Buffalmaccos Höllendarstellung im Camposanto in Pisa, die auch für die Bolognini-Kapelle in San Petronio maßgebend ist; dazu vor allem KLOTEN (wie Anm. 7), S. 84 ff. 
henden Mittelalters vom Inferno wieder, in dem alle Stände ebenso wie Päpste und Kaiser gleich behandelt werden.

Für den Propheten wurde unter den Verdammten keine eigene Ikonographie entwickelt. Er ist wie auch der Papst nackt und allenfalls sein Bart und das mit einer an kufische Zeichen gemahnenden Bordüre versehene Kopftuch signalisieren seine Fremdheit. Seine Identität ist ausschließlich anhand der Beschriftung zu erkennen. Für den Auftraggeber und den zeitgenössischen Betrachter ist der Prophet - sowohl in Pisa als auch in der Bolognini-Kapelle in Bologna - dazu instrumentalisiert worden, den angeblich abtrünnigen Papst der Schande preis zu geben. Das Skandalöse erwuchs aus dem Doppelbildnis und diente dazu, Nikolaus V. oder in Bologna die Gegner Johannes XXIII., die im Konzil von Pisa als Häretiker geschmähten Päpste Gregor XII. und Benedikt XIII., als Kirchenspalter zu diffamieren. ${ }^{11}$ Diesen schismatischen Gegnern galt das Interesse und sie sollten - den Topos des Niccolò und des Propheten aufgreifend - in effigie in die Hölle verdammt werden. ${ }^{12}$ Nicht die Charakterisierung der Gestalten lässt die Malerei so blasphemisch erscheinen, wobei die Zeitgenossen im 15. Jahrhundert diese 'Schandmalerei' durchaus in ihrer skandalisierenden Absicht verstanden, allerdings selbstverständlich in Bezug auf die Darstellung der innerkirchlichen Thematik, sondern der Kontext, die prominente Stellung und die namentliche Auszeichnung der Gestalten. Diente die Verurteilung im Bild den Zeitgenossen zur Identitätsbeschwörung der Anhängerschaft des bolognesischen Papstes Johannes XXIII., so wurde die 'Schande' im Bild von der relativ kleinen muslimischen Gruppierung in Italien 600 Jahre später in derselben Weise interpretiert und auch instrumentalisiert.

Das Beispiel der Bolognini-Kappelle soll deutlich machen, dass bis weit ins 15. Jahrhundert hinein nicht die mimetische Darstellung des Propheten oder der Muslime, gar in historisierender Absicht ${ }^{13}$ vorgetragen, zu erwarten ist, sondern ein Imaginaire der Muslime. Eine größere Realitätsnähe oder Ferne der Dargestellten sagt noch nichts über deren Wertschätzung, Verständnis, positive oder negative Konnotation aus. Allein der Kontext übermittelt in der Regel die Bildintention, wobei gerade diese - wie am Beispiel der Bolognini-Kapelle offensichtlich wird - je nach Rezipient, historischen Umständen und Bilderwartung ganz unterschiedliche Interpretationen findet. Allerdings besteht wohl kein Zweifel an der bereits bei der Entstehung angestrebten polemischen Ausrichtung der An-

11 Zur besonderen Verbindung des Auftraggebers Bartolomeo Bolognini zu dem im Konklave in Bologna 1410 gewählten Johannes XXIII., der sich seinerseits als Nachfolger Johannes XXII. verstand, dem das Pisaner Schandbild zugedacht war, KLOTEN (wie Anm. 7), S. $13-15$.

12 KLOTEN (wie Anm. 7), S. 86 f.

13 Eine eigenartige Form eines kulturellen Missverständnisses lässt sich in einer Zusammenstellung der Bilder des Propheten zum Karikaturenstreit beobachten. Alle historischen Darstellungen werden als nicht 'realistisch' kommentiert: Mohammed Image Archive. Depictions of Mohammed Throughout History, URL: <http://radiobergen.org/terrorism/ Mohammed Image Archive.htm> (7.2.2009). 
ordnung, die den Propheten und den Papst zentral unmittelbar über Luzifer in ihrer Qual zur Schau stellt.

\section{Der Wandel der Bedeutung des topischen Sarazenen}

Geradezu verblüffend ist die Erfahrung, wie topisch die Bildmotive sind, mit denen das Spätmittelalter in der Regel Muslime charakterisiert. Dies ist umso erstaunlicher, als die Kontakte in der Zeit der Kreuzzüge, aber auch durch Handel, Delegationen und Pilgerreisen relativ eng waren und insbesondere in Venedig, dem großen Tor in die Welt des Nahen und Fernen Ostens, die Künstler durchaus Muslime mit eigenen Augen sehen konnten. Nun kann man freilich, wie bereits das Beispiel der Cappella Bolognini zeigte, auch im 15. Jahrhundert nicht unbedingt eine mimetische Schilderung etwa osmanischer oder mameluckischer Persönlichkeiten erwarten, beginnt doch in dieser Zeit gerade eben erst das Interesse an der Darstellung der Besonderheit von Individuen, wie beispielsweise die Geschichte des frühen Porträts seit dem zweiten Drittel des 14. Jahrhunderts erkennen lässt. Nicht das Abbild des 'realen' Muslims wird denn in Malerei und Graphik des ausgehenden Mittelalters zu suchen sein, sondern die Darstellungen werden in unterschiedlichen Zusammenhängen jeweils andere Aspekte des Imaginaire des Orientalen verdeutlichen. Im Folgenden sollen einzelne Varianten angesprochen werden, um anhand der kontextuellen Situierung - sowohl innerhalb des Objektes als auch von den Interessen der Auftraggeber und Rezipienten her gesehen - das Spektrum der jeweiligen Deutungsangebote darzustellen.

In den literarischen und bildlichen Beschreibungen des 'Ostens' spielen neben den aus der Antike abgeleiteten Vorstellungen der unterschiedlichen Ethnien und Rassen ${ }^{14}$ die Sarazenen als Oberbegriff für alle nichtchristlichen, heidnischen, vor allem aber islamisch-östlichen Gruppierungen die entscheidende Rolle. Unter diesem Oberbegriff verbirgt sich nicht nur ein das ganze Mittelalter andauernder Diskurs, der jeweils mit anderen Feindbildern arbeitet, sondern er meint nicht selten auch den Feind in den eigenen Reihen. Schon sehr früh setzt sich die Vorstellung durch, Mohammed sei nichts anderes als der Gründer einer fehlgeleiteten Sekte und der Islam eine Häresie des Christentums, die mehr oder minder einsichtig einer Bekehrung gegenüber stünde. Insofern können mit Sara-

14 RUTH BaRTAL: The Image of the Oriental. Western and Byzantine Perceptions. In: East Meets West. Art in the Land of Israel. Hg. von Asher OVADIAH und NURITH KenNaNKedar. Tel Aviv 1998 (Assaph, Section B. Band 3), S. 131-148, bes. 131 - Debra HigGS STRICKLAND: Saracens, Demons, and Jews. Making Monsters in Medieval Art. Princeton (NJ) / Oxford 2003, S. 30-46. 
zenen durchaus auch Juden, Katarer und Waldenser umschrieben werden. ${ }^{15} \mathrm{Sa}-$ razenen stehen somit stellvertretend für die zu bekämpfenden innerkirchlichen Entwicklungen oder gar gesamthaft für mangelnde, fehlgeleitete Glaubenskraft. Da Juden und Muslime als eng miteinander verbunden verstanden wurden, soll doch Mohammed seine Lehre von jüdischen Gelehrten übermittelt bekommen und diese dann übel abgewandelt haben, wie vor allem Petrus Alfonsi und Petrus Venerabilis argumentieren, ${ }^{16}$ werden Muslime und Juden zumindest im 12. und auch noch im 13. Jahrhundert häufig nicht weiter differenziert. Ebenso können sich auch Mongolen und andere Völkerschaften unter dem zum Synonym für alle paganen Gesellschaften gewordenen Bild des Sarazenen finden. ${ }^{17}$ Zwar hat Geraldine Heng auf die zunehmende Kodifizierung des Lebens, des Rechts und damit auch der Differenzierung der Gruppen seit dem frühen 13. Jahrhundert aufmerksam gemacht und vor allem auf die im vierten Laterankonzil 1215 erlassenen Verordnungen hingewiesen, ${ }^{18}$ die eine visualisierte Differenzierung der Minderheiten durch spezielle Kleidervorschriften einfordern, aber darauf scheinen die Bilder selbst eher zögerlich zu reagieren. Es werden zwar unterschiedliche Formeln für orientalische Gestalten verwendet, ohne dass damit in der Regel jedoch konkrete ethnische Gruppen gemeint würden.

\section{a) Der Sarazene als gleichwertiger, ritterlicher Kämpfer}

Am weitesten verbreitet ist die Ikonographie des tapferen Kämpfers, der sich durch Habitus, oft abenteuerliche Rüstungen und Waffendetails von anderen Gruppen unterscheidet. In der nach 1333 in Paris, wohl im Atelier von Richard de Montbaston entstandenen Handschrift mit Werken der Reiseliteratur und dem Alexanderroman werden die üblichen Formeln für den kämpferischen Orientalen in mehreren Bildern für ganz unterschiedliche Parteien vom Nahen bis in den Fernen Osten gleichermaßen eingesetzt (Abb. 1). ${ }^{19}$

$15 \mathrm{Zu}$ den unterschiedlichen Gruppierungen JOHN VICTOR TOLAN: Sons of Ishmael. Muslims through European Eyes in the Middle Ages. Gainesville 2008, bes. S. IX-XIII und $46 \mathrm{ff}$.

16 Dazu TOlan (wie Anm. 15), S. 56 f.

17 STRICKLAND (wie Anm. 14), S. 192-206.

18 Geraldine HenG: The Romance of England. Richard Coer de Lyon, Saracens, Jews, and the Politics of Race and Nation. In: The Postcolonial Middle Ages. Hg. von JefFreY JEROME COHEN. New York (NY) 2000, S. 135-171, bes. 137 f.

19 Vgl. zur Handschrift Royal 19 D I der British Library, London CONSUELO WAGER DUTSCHKE: Francesco Pipino and the Manuscripts of Marco Polo's «Travels». Diss. University of California, Los Angeles (Ca) 1993 (ProQuest Dissertations \& Theses Full Text, Publication Number AAT 9320360), S. 338-346, Kat. Nr. 51. - Zum möglichen Atelier RICHARD H. und MARY A. ROUSE: The Commercial Production of Manuscript Books in Late-Thirteenth-Century and Early-Fourteenth-Century Paris. In: Medieval Book Production. Assessing the Evidence. Hg. von LINDA L. BROWNING. Los Altos Hills (Ca) 1990, S. 103-131, bes. 109-111. 


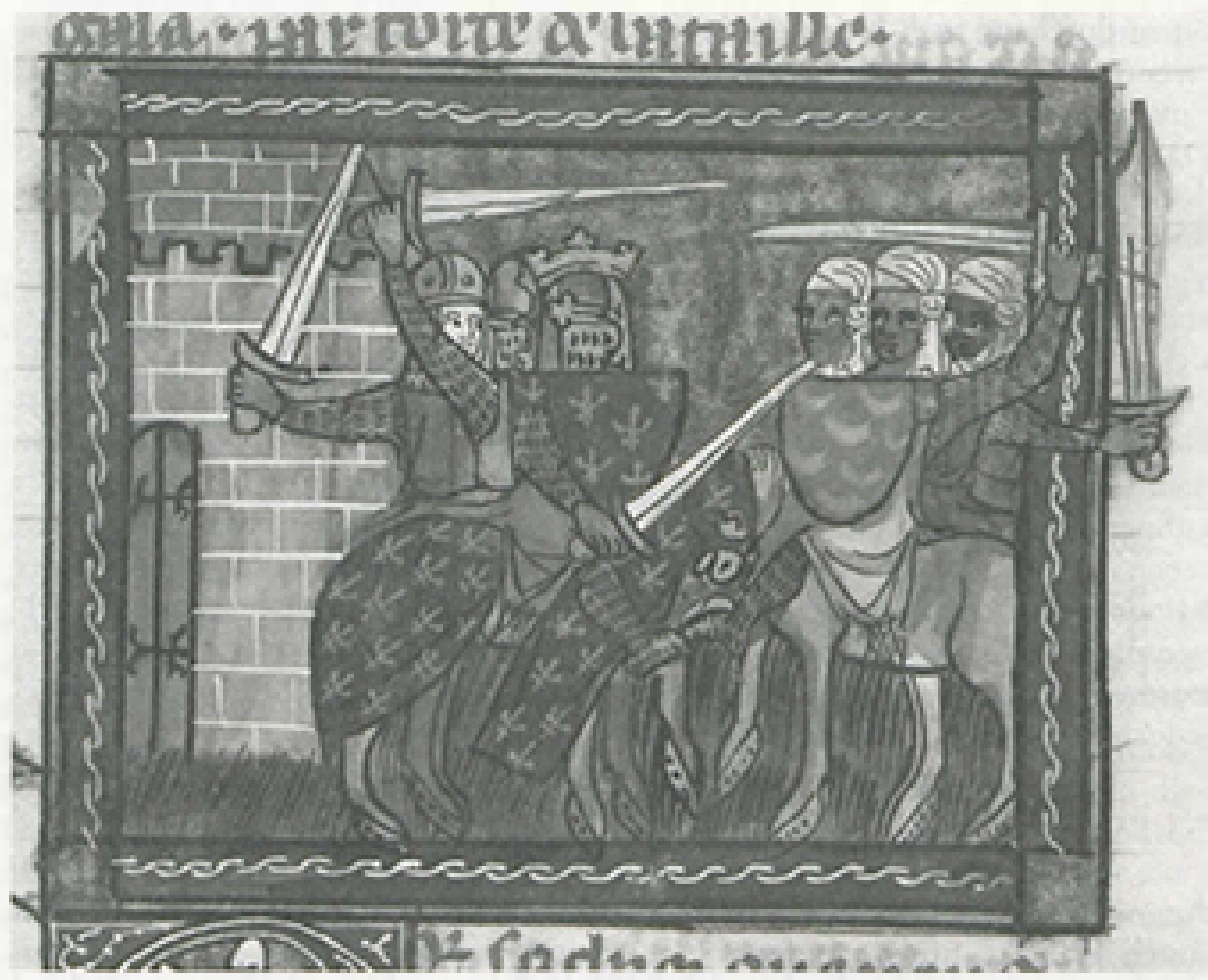

Abb. 1: Die Leute von Mien erheben sich gegen Kublai Khan. Reisehandschrift, Paris, nach 1333. London, The British Library: Royal 19 D I, fol. 103

Die gegen Kublai Khan sich erhebenden Aufständischen von Mien übernehmen den in der gesamten Handschrift für die 'Fremden', die Orientalen, verwendeten Typus des Sarazenen. Dieselben Formeln schildern auch den Kampf der französischen Kreuzfahrer mit den Sarazenen (Abb. 2). Hierzu gehören die beeindruckenden Krummschwerter, deren Bedrohlichkeit im Kreuzritterkampf durch die beiden aus dem Bild heraus in den Raum des Codex hinein ragenden Exemplare verstärkt wird. Gleichermaßen ist das um den Kopf gewundene, seitlich von einem einfachen Knoten zusammengefasste Tuch ein fester Bestandteil dieser Formel. Beide Parteien sind vollständig geschützt mit Ringelpanzern, über denen sie Waffenröcke tragen. Während aber die Partei Kublai Khans und der Kreuzritter durch den Helm mit dem heruntergelassenen Visier zusätzlichen Schutz genießt, der lediglich bei der hintersten Gestalt nicht so massiv wirkt, greift diese doch mit offenem Visier an, sind die orientalisierten Kämpfer mit bloßem Haupt angetreten und tragen lediglich den Turban. Der Erste aus der Schar von Mien und der Sarazenen ist denn auch bereits von einem Schwertstreich des mongolischen Heerführers beziehungsweise der Kreuzritter verletzt und holt gerade zum Gegenschlag aus. Die beiden Parteien sind jeweils spiegelbildlich angeordnet und 
lediglich der nur von einem Tuch geschützte Kopf und die martialischeren Waffen, vor allem das 'Sarazenenschwert', lassen die Kämpfer von Mien und die Sarazenen im Gegensatz zu den hier den westlichen Kämpfern gleichgesetzten Mongolen als üblichen Typus des 'Orientalen' erkennen.

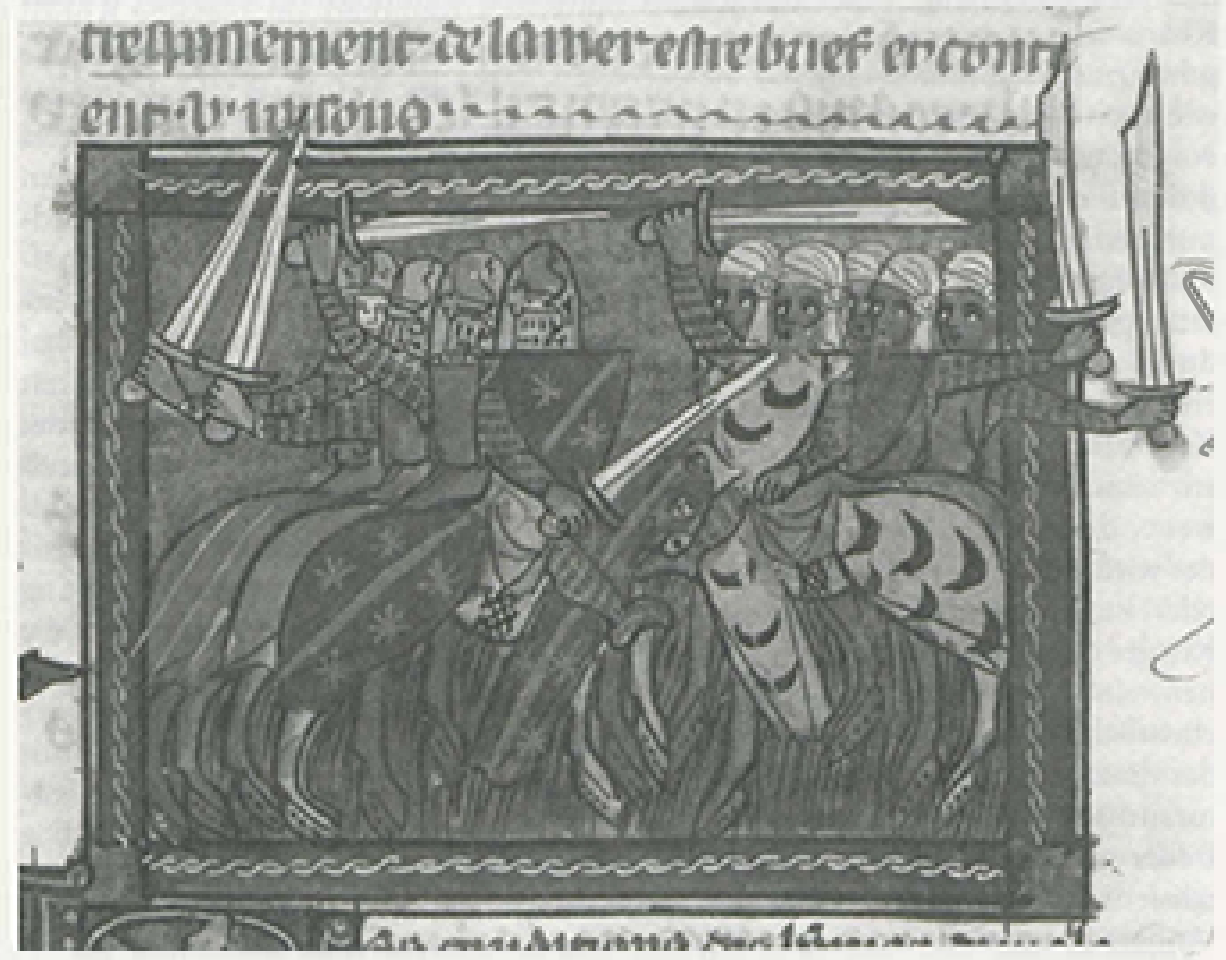

Abb. 2: Kampf zwischen Sarazenen und Kreuzrittern. Reisehandschrift, Paris, nach 1333. London, The British Library: Royal 19 D I, fol. 189v

Alle Gruppierungen gehören einem phantastischen Imaginaire an, in dem Fremdes und Eigenes auf unterschiedliche Weise verwoben ist, und dies obwohl zu den Mongolen eine durchaus breite Literatur existierte, die sich gerade für deren physische 'Schrecklichkeit' interessierte. ${ }^{20}$ Auffällig ist zunächst die bei allen Parteien den westlichen Gewohnheiten vollständig angepasste Bewaffnung und Rüstung sowie der Schutz der Pferde durch Schabracken. Lediglich die Kämpfer von Mien reiten auf ungeschützten Pferden, was freilich eher in den Bereich der Variation als der Charakterisierung gehört. ${ }^{21}$ Alle in diesem Band versammelten Akteure kriegerischer Auseinandersetzungen sind also im weiteren Sinn dem

20 STRICKI.AND (wie Anm. 14), S. 192-206, auch zur Gleichsetzung mit den Tartaren.

21 London, The British Library: Royal 19 D I, auf fol. 125 reiten die Kämpfer von Maabar auf Pferden, die von Schabracken geschützt sind. 
Körper des westlichen Rittertums eingegliedert. Freilich sind die Gewichte dennoch unterschiedlich verteilt, was allerdings mit heraldischen Mitteln erreicht wird. Am auffälligsten ist die Unterscheidung der Hautfarbe, sind doch die Leute von Mien wie auch die Sarazenen schwarz, während ihre Kontrahenten - und dies lässt das offene Visier des jeweils hintersten Kämpfers im Gefolge Kublai Khans wie auch unter den Kreuzrittern deutlich erkennen - Weiße sind. Die schwarzen Halbmonde auf der gelben Schabracke des Anführers der Sarazenen wie auch die gelben Halbmonde auf dem orangen-farbigen Schild des Anführers von Mien unterstützen ihre Zuordnung, während der Anführer der Mongolen durch die goldenen Lilien auf blauem Grund dieselbe noble heraldische Zuordnung erhält wie der französische König im Heer der Kreuzfahrer. ${ }^{22}$ Der sagenhafte Mongolenherrscher ist zu einem der französischen Krone äquivalenten Potentaten geworden. Der Angriff des Kreuzfahrerheers im Heiligen Land (Abb. 2) hat durch den goldenen Stern auf blauem Grund dieselbe auszeichnende Farbigkeit erhalten, verweist aber zugleich mit dem Stern auf die östliche Zugehörigkeit des Kreuzfahrerheers als Gesamtes. ${ }^{23}$ Die schwarze Hautfarbe jeweils einer der Parteien, nämlich der Sarazenen im weitesten Sinne, ist Teil der heraldischen Erzählweise, die in der Londoner Handschrift für sehr unterschiedliche Texte verwendet wird, und dient zunächst der Parteienbezeichnung, ohne damit eine Wertung oder konkretere Charakterisierung zu meinen. Die Leute von Mien und die Krieger aus dem Nahen Osten, denen die Kreuzfahrer im Heiligen Land begegnen, sind gleichermaßen Sarazenen. Der kriegerische Sarazene ebenso wie der christliche Orient sind somit ein weit entferntes Spiegelbild der eigenen Kultur, das diese nicht nur zum Maßstab macht, sondern die Anderen zu einer Art kulturanthropologischen Entwicklungsstufe der eigenen Kultur werden lässt. Die Bilder schaffen mit dieser Kombination von Fremdem mit Eigenem eine 'Orientalisierung ${ }^{24}$, die nicht so sehr der Abgrenzung dient als der Inferiorisierung des 'Anderen' auf eine noch der eigenen Kultur anzupassende Entwicklungsstufe. Zugleich verdeutlichen sie dem Betrachter die Ferne in einer ihm verständlichen Nähe.

22 London, The British Library: Royal 19 D I, fol. 187v: im Schiff, in dem die Kreuzfahrer die Sarazenenfestung belagern, sind um den französischen König weitere Parteien mit ihren heraldischen Zeichen versammelt.

23 FANNY CAROFF: Différencier, caractériser, avertir. Les armoiries imaginaires attribuées au monde musulman. Médiévales 38 (2000) S. 137-147, bes. 139.

24 IVAn Davidson Kalmar: Jesus Did Not Wear a Turban. Orientalism, the Jews, and Christian Art. In: Orientalism and the Jews. Hg. von IVAN DAVIDSON KALMAR und DEREK JONATHAN PENSLAR. Hanover / London 2005 (The Tauber Institute for the Study of European Jewry Series), S. 3-31 und 225-228, bes. 9 f., betont ebenfalls, dass diese Varianten einer Orientalisierung im 13. Jahrhundert bereits als frühe Form des Orientalismus verstanden werden können. 


\section{b) Der Sarazene als Berserker}

Noch deutlicher - allerdings mit einer anderen Note - wird diese rein imaginative Zusammenstellung der Sarazenen in der verwandten Handschrift der «Histoire d'Outremer» des Wilhelm von Tyrus gestaltet. ${ }^{25}$ Das 1337 datierte Manuskript dürfte aus demselben Umkreis stammen wie die Londoner Reisehandschrift, aus der Pariser Montbaston-Werkstatt und deren Nachbarn Geoffroy von Saint-Léger. ${ }^{26}$ Die Schlacht um Damaskus (Abb. 3) arbeitet mit denselben Mitteln wie die Kampfszenen in den Londoner Reiseerzählungen. Allerdings werden in diesen Bildern die Sarazenen nun eindeutig mit einer bestimmten Wertung dargestellt. Während die Gesichter in der Londoner Handschrift mit ihren riesig aufgerissenen Augen (Abb. 1 und 2) denjenigen der Weißen entsprechen und lediglich schwarz gefärbt sind, haben sie in der "Histoire d'Outremer» karikierende Züge gewonnen. Der grimmige Ausdruck, die ausgeprägt knolligen Nasen und das gekrauste Haar schaffen eine negativ konnotierte Variante der Schwarzen, die gleichwohl ambivalent bleibt. Ihre Attacke reiten die Sarazenen mit einer bloßen Tunika bekleidet mit nackten Füßen in den Steigbügeln ohne jegliche Rüstung. Das Berserkerhafte, das diesen Gegnern der Kreuzfahrer im Gegensatz zu denjenigen der Londoner Handschrift aneignet, schwankt zwischen Schrecken und Bewunderung, erhält aber durch die Heraldik eine eindeutige Erklärung. Die beiden hinter dem Anführer reitenden Sarazenen tragen Schilde, auf denen einmal ein schwarzer Drache und im anderen Fall zwei schwarze Hände zu sehen sind. Der schwarze Drache, ein häufiges Motiv zur Bezeichnung von Muslimen, wird als Kreatur oder sogar Synonym des Teufels verstanden und denunziert somit die zugleich bewunderte Kampfeswut der Gegner als satanische Kraft. ${ }^{27}$

Hatten die Kreuzfahrerhandschriften im 13. Jahrhundert besonderen Wert auf die Nobilitierung ihrer eigenen Leistung durch eine Angleichung ihres Gegners gelegt, sind die bereits mit einer gewissen Nostalgie behafteten Kreuzfahrertexte im 14. Jahrhundert in einem ganz anderen Milieu und mit veränderten Interessen illustriert worden. Insbesondere der erste Valoiskönig Philipp VI. verknüpfte seine Bestrebungen, sich in direkter Linie der Kapetinger zu verstehen, eng mit einer historischen Legitimation, die nicht nur in dem enormen Zuwachs der historischen Chroniken zum Ausdruck kommt, sondern auch in seiner Bemühung, eine neue Kreuzzugspolitik zu propagieren. ${ }^{28}$ In diesem Kon-

25 Paris, BnF: Ms. fr. 22495; dazu JAROSLAV THAYER FOLDA: The Illustrations in Manuscripts of the "History of Outremer" by William of Tyre. 3 Bde. Diss. Baltimore 1968 (Mikrokopie Ann Arbor [Mi] 1973), Bd. 2, S. 176-203.

26 Colette Beaune: Les manuscrits des rois de France au Moyen Âge. Le Miroir du Pouvoir. Paris 1997 (Bibliothèque de l'Image), S. 182: in der Werkstatt des Geoffroy von Saint-Léger entstanden (Richard H. und Mary A. Rouse).

27 Dazu Caroff (wie Anm. 23), S. 142 f. - Strickland (wie Anm. 14), S. 180 f.

28 ANNE D. HEDEMAN: The Royal Image. Illustrations of the "Grandes Chroniques de France» 1274-1422. Berkeley (Ca) u. a. 1991 (California Studies in the History of Art. Band 28), S. 63-68. 


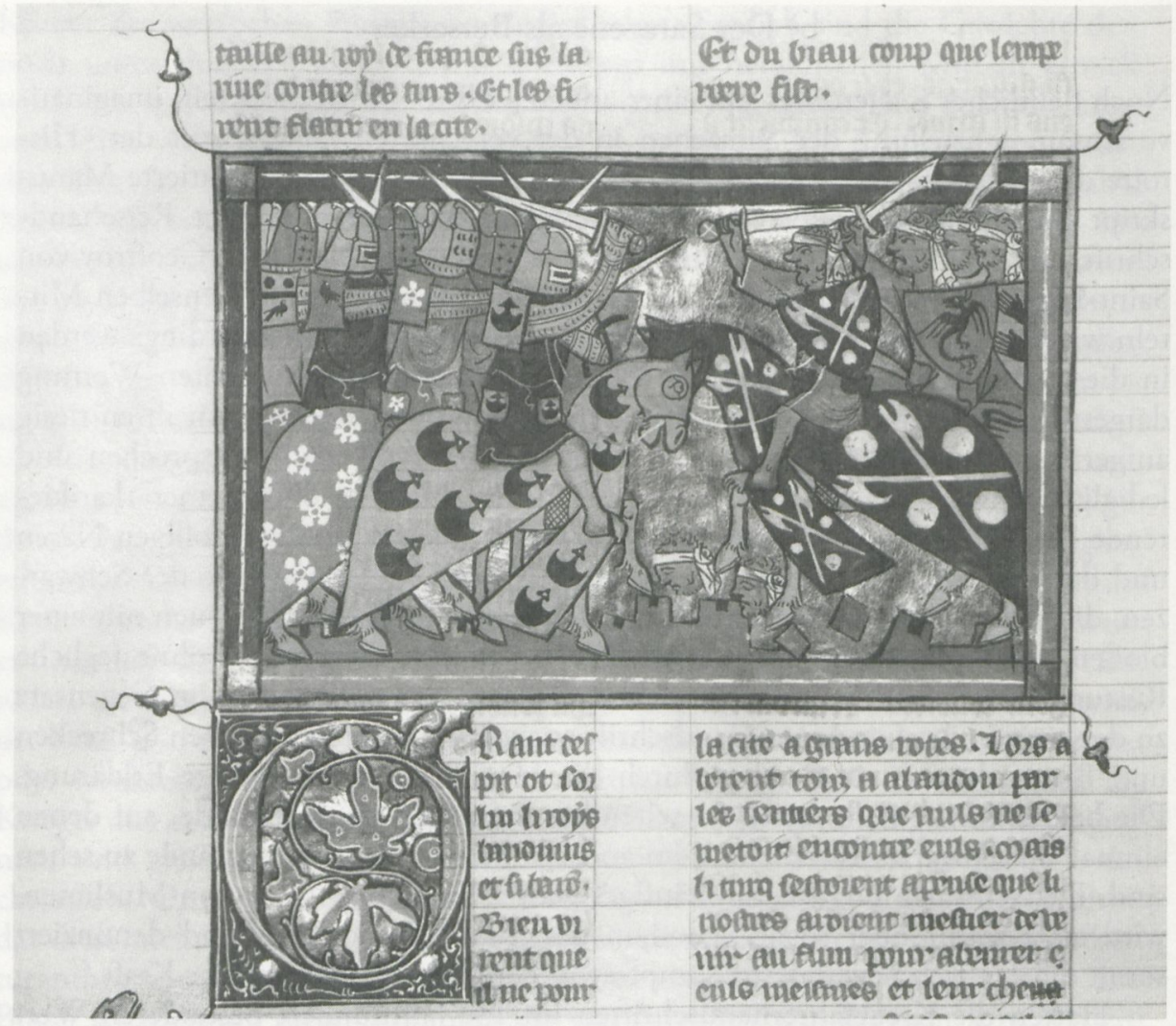

Abb. 3: Die Schlacht um Damaskus. Wilhelm von Tyrus, "Histoire d'Outremer», Paris, 1337. Paris, Bibliothèque nationale de France: Ms. fr. 22495, fol. 154v

text, in dem auch die Auseinandersetzung mit England eine Rolle spielt, ${ }^{29}$ geht es vor allem darum, Philipps direkte Nachfolge des Heiligen Ludwig zu beschwören. Hierzu dienen eine Reihe historischer Handschriften, unter denen auch Texte wie die "Histoire d'Outremer» in auffällig vielen Exemplaren produziert werden. ${ }^{30}$ Der betont berserkerhaften, ja teuflischen Kampfeswut der Sarazenen in der Pariser Handschrift (Abb. 3) steht umso vorteilhafter das angemessene und vor allem disziplinierte, also 'kultivierte' Verhalten der Kreuzritter gegenüber: Sie kämpfen für eine gerechte Sache gegen vom Satan verleitete Gegner. In diesem Bild wird das fortgesetzt, was wir schon in der Londoner Reisehandschrift be-

29 Philipp VI. sammelt ab 1334 für einen Kreuzzug und benutzt dieses Geld 1335-1337 zur Finanzierung des Krieges gegen England; dazu HedEMAN (wie Anm. 28), S. 64.

30 FolDA (wie Anm. 25), Bd. 1, S. 402-477, stellt eine große Gruppe von vergleichbaren Handschriften zusammen, die alle durch ein ausgeweitetes Bildprogramm und ähnliche Entstehungsumstände auffallen. 
obachteten, eine Konkretisierung der Kreuzfahrer und nun negative Imaginierung der Sarazenen. Die Rüstungen, Helme und die von den Kreuzfahrern getragenen Achselstücke mit den Wappen sind im Vergleich zu der phantastischen Schilderung der angreifenden Sarazenen von einem ganz anderen Realitätsgrad.

Die in der Pariser Handschrift angelegte Unterstellung einer vom Teufel inspirierten sarazenischen Kampfeswut ist bereits im Text Wilhelms von Tyrus vorgeformt. ${ }^{31}$ Die mimetische Angleichung der sarazenischen Gegner an 'wilde Schwarze' ist freilich nicht ethnisch zu lesen, sondern schöpft aus der Vorstellung dämonisierter Fremder, die in die Regionen der außerchristlichen Welt gehören. ${ }^{32}$ Jeffrey Jerome Cohen verweist auf die in den "Grandes Chroniques» mit dämonischen Masken vor Córdoba gegen das Heer Karls des Großen antretenden Sarazenen und wertet dies als Ausdruck der Reflexion über das 'Sarazenische', das durchaus auch als christliches Konstrukt verstanden worden sei. ${ }^{33}$ In dem für Charles V. hergestellten Exemplar der "Grandes Chroniques de France» wird diese Textstelle auch ins Bild umgesetzt, und die in den anderen Darstellungen lediglich als schwarze Pendants zu den christlichen Rittern erscheinenden Gegner tauchen hier vor dem eher verdutzt als erschreckt wirkenden Karl mit dämonischen Masken auf. ${ }^{34}$ Dem Zeitgenossen muss diese künstliche Verfremdung verständlich gewesen sein, kannte er doch ähnliche Masken etwa vom Charivari. ${ }^{35}$ Die Wandelbarkeit des Sarazenenbildes ist also durchaus bewusst geblieben, so dass es mit den in diesen Darstellungen zum Ausdruck gebrachten negativen Vorurteilen keineswegs als festgeschrieben gelten kann.

\section{Heiden - Juden - Osmanen: eine 'orientalisierende' Überblendung}

Die vorgestellte Formel für den Sarazenen wurde nicht aus einem neuen Repertoire kreiert, es handelt sich vielmehr um eine synthetische Gestalt: In ihm scheint das Bild des Heiden, manchmal auch des antiken Kriegers und nicht selten des Juden auf. Die Frage, wie weit die historische und geographische Konkretisierung der Erzählung in der Zeit vor 1400 ein Darstellungskriterium ist, kann wohl nicht summarisch beantwortet werden. Der Sarazene ist meist nicht nur in seiner Wertung ausschließlich über den Kontext lesbar, sondern sogar in

31 STRICKLAND (wie Anm. 14), S. 169.

32 JefFrey Jerome Cohen: On Saracen Enjoyment. Some Fantasies of Race in Late Medieval France and England. Journal of Medieval and Early Modern Studies 31/1 (2001) 113-146, bes. 115-122 mit weiterer Literatur.

33 COHEN (wie Anm. 32), S. $121 \mathrm{f}$.

34 Paris, BnF: Ms. fr. 2813, fol. 119; Abb. Strickland (wie Anm. 14), Abb. 80 und S. $169 \mathrm{f}$.

35 Paris, BnF: Ms. fr. 146, fol. 34; Abb. ChARLES STERLING: La peinture médiévale à Paris 1300-1500. Bd. 1. Paris 1987, Abb. 23. 
seiner Zuordnung zu einer bestimmten ethnischen Gruppe. In der so genannten Arsenal-Bibel ${ }^{36}$, einer Handschrift, die zwischen 1250 und 1254 in der letzten Hochburg der Kreuzfahrer, in Akkon, als altfranzösische Bibel hergestellt wurde, nehmen die einzelnen Volksgruppen einen geradezu symbiotischen Gemeinschaftscharakter an. Wahrscheinlich im Auftrag des französischen Königs entstanden, werden die Juden - etwa in Moses Rede an das Volk - mit den phrygischen Mützen der Antike als allgemein fremdländisch gekennzeichnet. ${ }^{37}$ Die israelitischen Kämpfer tragen konische Helme mit einem nach vorne ausschwingenden Grat, die der antikischen Version der phrygischen Mütze entsprechen. ${ }^{38}$ Dem mittelalterlichen Verständnis einer antikischen Rüstung folgen auch der auf einem Lederwams befestigte Schienenpanzer und die Achsel und Unterleib schützenden vergoldeten, schmalen Schienen, die in der Arsenal-Bibel verschiedentlich vorkommen. Die alttestamentlichen Kämpfer sind somit in dieser für den französischen Hof hergestellten Handschrift von Akkon gleichsam kolonialisiert, indem sie als biblische Vorläufer des zeitgenössischen Ritters verstanden werden und zugleich aufgehen in jener allgemein orientalisch-antikischen Kultur, in welcher die Kreuzfahrer das christianisierte Heilige Land sehen und die, so belegte Daniel Weiss eindrucksvoll, der königlichen Ideologie einen adäquaten Ausdruck verlieh. ${ }^{39}$

\section{a) Orientalisierung und Ambivalenz}

Gleichwohl scheint das 'multikulturelle' Ineinanderübergehen der unterschiedlichen Gruppen nicht ganz so wertfrei übermittelt zu werden, wie man zunächst glauben möchte. Im Frontispiz zum Buch Judith (Abb. 4) reitet das assyrische Heer des Holofernes mit den Bannern des Orients - Halbmond und Stern - als westlich gerüstete Schar gegen Bethulien. Nur wenig deutet darauf hin, dass es sich nicht um die übliche ritterliche Schar handeln könnte, wie sie etwa auch die alttestamentlichen Reiterheere der ebenfalls in königlichem Auftrag entstandenen Morgan Bibel vertreten. ${ }^{40}$ Über dem Ringelpanzer, dessen Kapuze sie über den Kopf gezogen haben, tragen die Kämpfer einen Eisenhut. Die Dreieckschilde und ein geschürzter Waffenrock entsprechen der üblichen Rüstung westlicher

36 Paris, BnF: Ms. Arsenal 5211; dazu Daniel H. WeIss: Art and Crusade in the Age of Saint Louis. Cambridge 1998, S. 85-104 - Derselbe: Biblical History and Medieval Historiography. Rationalizing Strategies in Crusader Art. Modern Language Notes 108 / 4 (1993) S. 710-737, bes. 715 f. - HugO BuCHTHAL: Miniature Painting in the Latin Kingdom of Jerusalem. Oxford 1957, S. 54-68.

37 Abb. Buchthal (wie Anm. 36), Taf. 66a.

38 Abb. Buchthal (wie Anm. 36), Taf. 66a, 67a etc.

39 WEISS, Art and Crusade (wie Anm. 36), S. 203.

40 New York, The Pierpont Morgan Library: M 638, fol. 20v und fol. 37v; dazu SYDNEY C. Cockerell: Old Testament Miniatures. A Medieval Picture Book with 283 Paintings from the Creation to the Story of David. London 1969, Abb. 133, 170. 


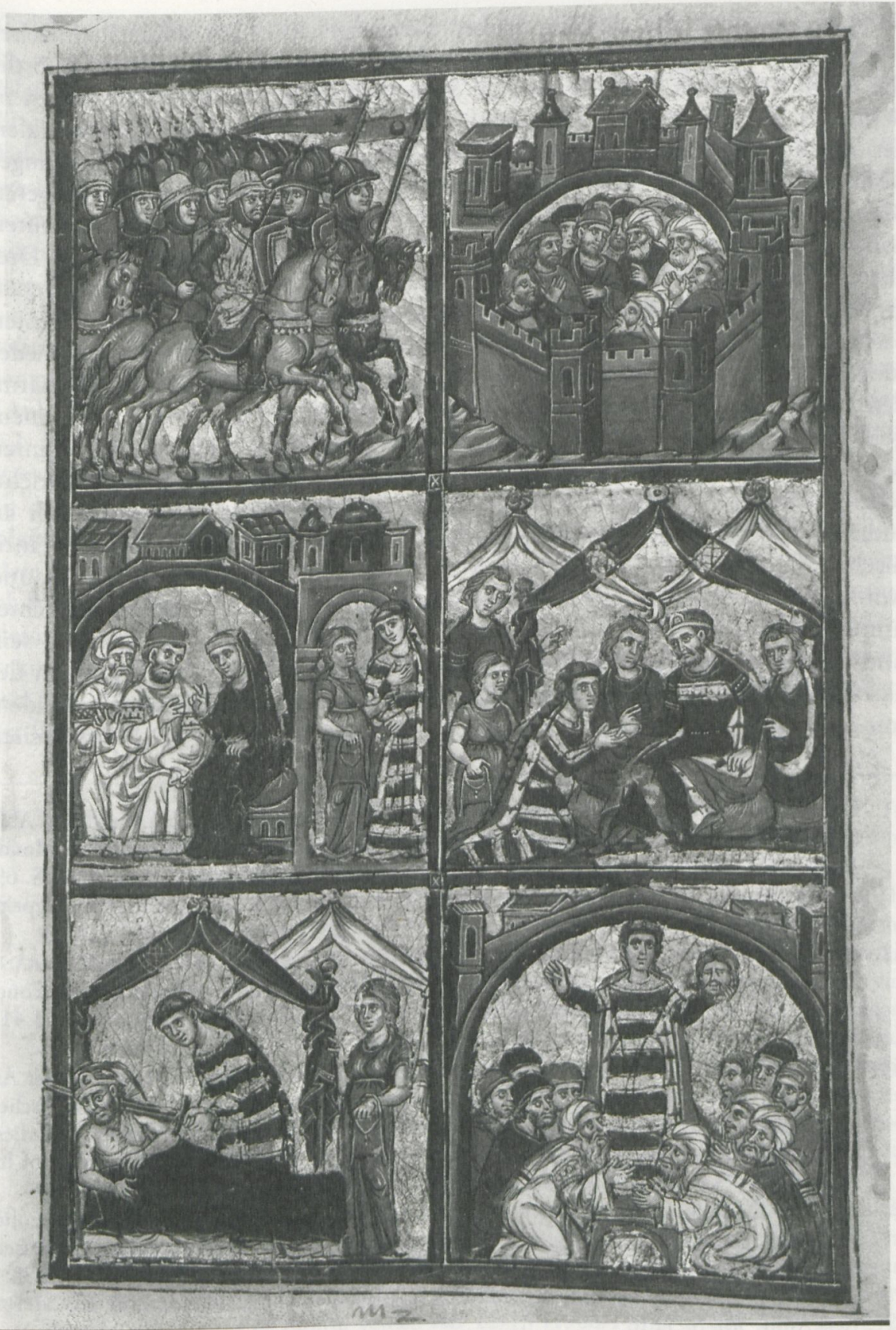

Abb. 4: Frontispiz zum Buch Judith. Französische Bibel, Akkon, 1250-1254. Paris, Bibliothèque nationale de France: Ms. Arsenal 5211, fol. 252 
Kämpfer, lediglich die auffällig kurzen Steigbügel, die den vordersten Reiter beinahe mit angezogenen Beinen auf dem Pferd sitzen lassen, gemahnen an östliche Gepflogenheiten. ${ }^{41}$ In der zweiten Szene beratschlagen sich die Israeliten in Bethulien. Die meisten der Männer sind in eine rote oder blaue Tunika gekleidet, nur dem im Zentrum sitzenden jungen Mann liegt noch ein Pallium ungewöhnlicherweise über der rechten Schulter. Außerdem tragen alle jüngeren Männer die sowohl in byzantinischen als auch orientalischen Handschriften vorkommende, an einen Fes erinnernde krempenlose Kopfbedeckung. ${ }^{42}$ Drei ältere Männer sind mit weißen Bärten und zu einem Turban geschlungenen großen Tüchern als islamische Würdenträger gekennzeichnet. In der nächsten Szene spricht Judith in westlicher Witwengewandung zu den Ältesten, die wiederum in ihren weißen Gewändern mit breiten goldenen Bändern am Oberarm, dem Tiraz, als aristokratische Muslime gekennzeichnet sind. ${ }^{43}$ Die mit einem gold-blau gestreiften Gewand und einem bodenlangen, mit Pelz verbrämten Mantel bekleidete Judith, die mit der Magd ins Lager des Holofernes aufbricht, scheint zunächst westlich gekleidet, ebenso wie die Magd. Ungewöhnlich an Judith ist die für Frauen unübliche Farbigkeit, die aber wiederum an die byzantinischen Vorlagen erinnert, die den Malern offenbar ebenso wie westliche und wohl auch orientalische zur Verfügung standen. ${ }^{44}$ Für den westlichen Betrachter unmissverständlich freilich muss diese Gewandung pejorativ zu verstehen sein und zumindest auf Judiths List verweisen. ${ }^{45}$ Holofernes, vor dem Judith in der anschließenden Darstellung kniet, trägt ein vereinfachtes byzantinisches Diadem mit einer aufgesetzten bogenförmigen Platte über der Stirn. ${ }^{46}$ Die mit breiten

41 Dazu etwa die Darstellung eines Ayyoubidischen Reiters des 13. Jahrhunderts; Abb. L'Orient de Saladin. L'art des Ayyoubides. Ausstellungskatalog Paris, Institut du Monde Arabe 2001 / 2002. Hg. von SOPHIE MAKARIOU und ERIC DelPONT. Paris 2001, S. 69. - Allgemein H. Russell Robinson: Oriental Armour. New York (NY) 1967, Reprint Mineola (NY) 2002, S. 11.

42 Die Mützenform ist wohl aus dem Orient nach Byzanz gelangt; dazu MARIA G. PARANI: Reconstructing the Reality of Images. Byzantine Material Culture and Religious Iconography (11th - 15th Centuries). Leiden 2003 (The Medieval Mediterranean. Band 41), S. $70 \mathrm{f}$.

43 WeISS, Art and Crusade (wie Anm. 36), S. 184. - Die Goldauszeichnungen an der Ärmeltunika kennt auch das byzantinische Gewand, wohl seinerseits an den orientalischen Tiraz angelehnt; PARANI (wie Anm. 42), S. 54. - Im Madrider Skylitzes kommt diese Gewandung sehr häufig vor; Abb. VASILIKI TSAMAKDA: The Illustrated Chronicle of Ioannas Skylitzes in Madrid. Leiden 2002, Abb. 85.

44 Vgl. die Darstellung der Tänzerinnen in der Handschrift Rom, Biblioteca Apostolica Vaticana: Vat. gr. 752, fol. 448v; Abb. AXINIA DŽUROVA: Byzantinische Miniaturen. Schätze der Buchmalerei vom 4. bis zum 19. Jahrhundert. Regensburg 2002, Taf. 62. Zur Amalgamierung der unterschiedlichen Vorlagen in dieser Handschrift BUCHTHAL (wie Anm. 36), S. $54 \mathrm{ff}$.

45 Dazu Michel Pastoureau: Des Teufels Tuch. Eine Kulturgeschichte der Streifen, Frankfurt am Main / New York 1995, S. 20 f.

46 Parani (wie Anm. 42), Taf. 31c. 
Goldstreifen über der Brust und schmalen Clavi an den Ärmeln dekorierte Tunika scheint ein Gewandtypus zu sein, der sich etwa auch in den von westlichen Malern hergestellten Illustrationen des Skylitzes ähnlich findet und wohl eine Umsetzung byzantinischer Gewänder meint. ${ }^{47}$ Der über die Schulter geworfene Fehmantel hingegen, vor allem aber die übereinander geschlagenen Beine sind westliche Motive. ${ }^{48}$ Die Fremdheit des Ganzen wird schließlich in der letzten Szene mit der Darstellung zu Judiths Triumph besonders offensichtlich. Auf einem Schemel stehend präsentiert sie frontal den Kopf des Holofernes und ragt mit ihren weit ausgebreiteten Armen wie eine Statue vor den Bethuliern auf, die sich adorierend vor ihr auf die Knie geworfen haben. Hier beherrschen die orientalisierten Würdenträger die Gruppe der Adoranten und gleichen nun deutlich jenen hohen Amtsträgern, die auch in einer syrischen Handschrift vorkommen (Abb. 5$)^{49}$. In dieser Form ist die Szene von Judiths Triumph einmalig. Seit dem 12. Jahrhundert hat sich die Judithikonographie zu einer Tugendikonographie entwickelt, ${ }^{50}$ wird Judith doch als Inbegriff der Castitas gefeiert. Die Darstellung in der Arsenal-Bibel freilich scheint einen Diskurs aufzugreifen, der ambivalent bleibt. In der Bibel von Roda (Abb. 6) ${ }^{51}$, einer spanischen illustrierten Bibel aus dem 11. Jahrhundert, findet sich ein Vorläufer, der dem westlichen Zeitgenossen eher verständlich gewesen wäre, wird doch dort Judith von den Bethuliern mit dem antiken Akklamationsgestus begrüßt. Das Bild in der Arsenal-Bibel jedoch rekurriert auf den Typus des triumphierenden, erhöhten byzantinischen Kaisers, vor dem die Feinde in Proskynese fallen. Ähnlich ist dieses Zeremoniell im Psalter Basilius II.

47 Madrid, NB: Codex Vitr. 26-2, fol. 106; Abb. TSAMAKDA (wie Anm. 43), Abb. 239.

48 New York, The Pierpont Morgan Library: M 638, fol. 40v; Abb. COCKERELL (wie Anm. 40), Abb. 247.

49 Istanbul, Bibliothek des Topkapi Sarayi Müzesi: Ahmet III 1227, fol. IV und 2r: Es handelt sich um ein Diptychon, auf dessen einer Seite Gelehrte oder Studenten dem auf der rechten Seite thronenden Autor Dioskorides Abschriften seines Textes darbringen. Die Bilder entsprechen byzantinischen Autorenbildern, wie sie auch in Evangelien vorkommen, allerdings sind die Gestalten in der Kleidung islamisiert. Auf der nachfolgenden Seite (fol. 2v), auf der sich der Autor mit einem Studenten unterhält, sitzen beide mit untergeschlagenen Beinen auf einem runden Kissen, was als Hinweis auf ihre islamische Herkunft verstanden werden darf. - Abb. The Glory of Byzantium. Art and Culture of the Middle Byzantine Era A. D. 843-1261. Ausstellungskatalog Metropolitan Museum of Art, New York. Hg. von Helen C. Evans und William D. WiXOM. New York (NY) 1997, Nr. 288, S. 429-433.

50 Leslie Abend Callahan: Ambiguity and Appropriation. The Story of Judith in Medieval Narrative and Iconographic Traditions. In: Telling Tales. Medieval Narratives and the Folk Tradition. Hg. von Francesca Canadé SAUtman, Diana Conchado und GiUSEPPE CARLO Di SCIPIO. New York 1998, S. 79-99, bes. 84-86 - Mira FriedmaN: The Metamorphoses of Judith. Jewish Art 12/13 (1986/1987) S. 225-246, bes. 232 235.

51 Paris, BnF: Ms. lat. 6, fol. 134v; dazu FrANCES G. GODWIN: The Judith Illustration of the Hortus Deliciarum. Gazette des Beaux-Arts 36 (1949) S. 25-46, dort Abb. 5. 


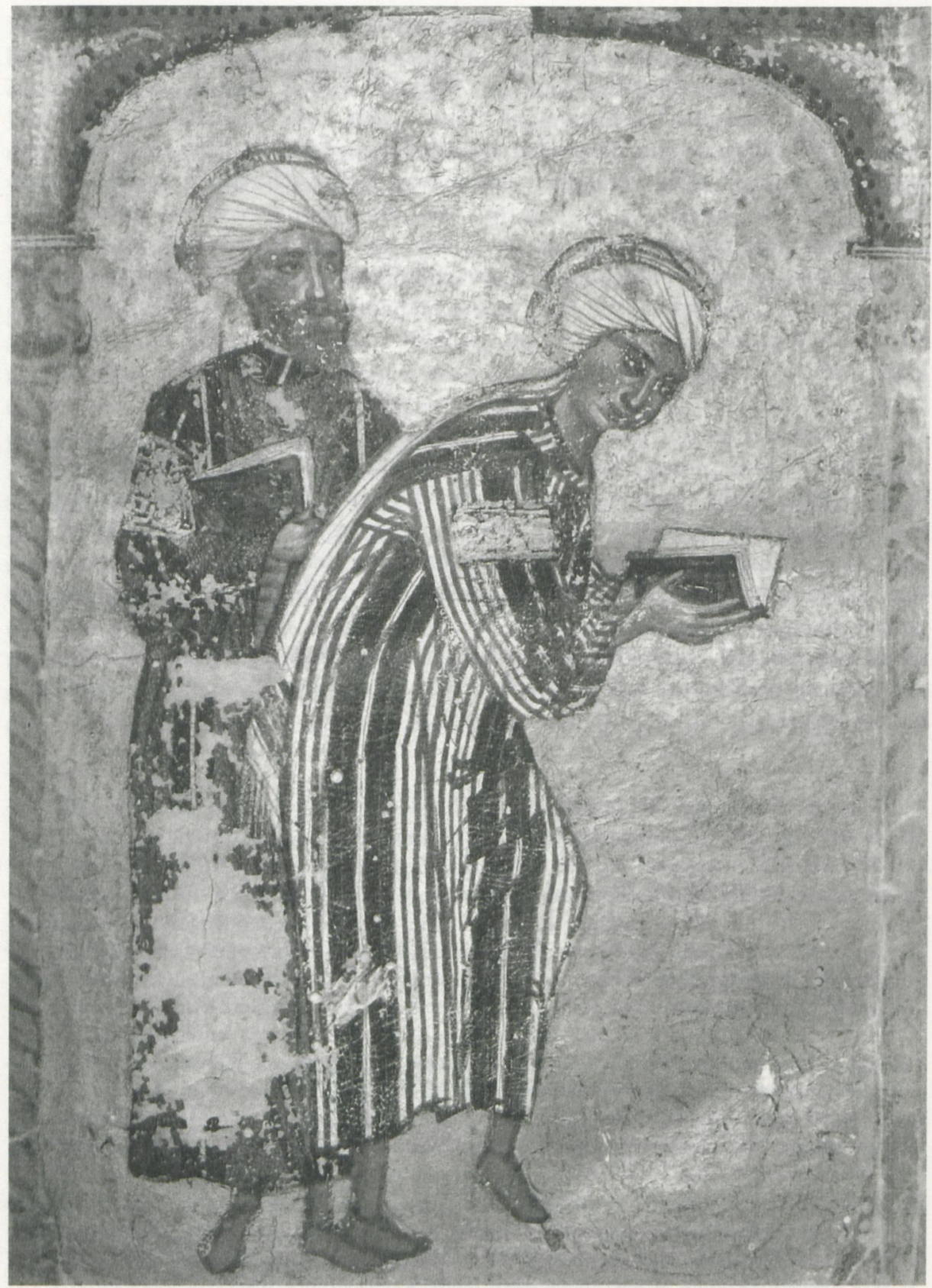

Abb. 5: Gelehrte präsentieren Dioskorides das Buch. "De materia medica», Nord-Mesopotamien, 1229. Istanbul, Bibliothek des Topkapi Saray Müzesi: Ms. Ahmet III 1227, fol. IV 


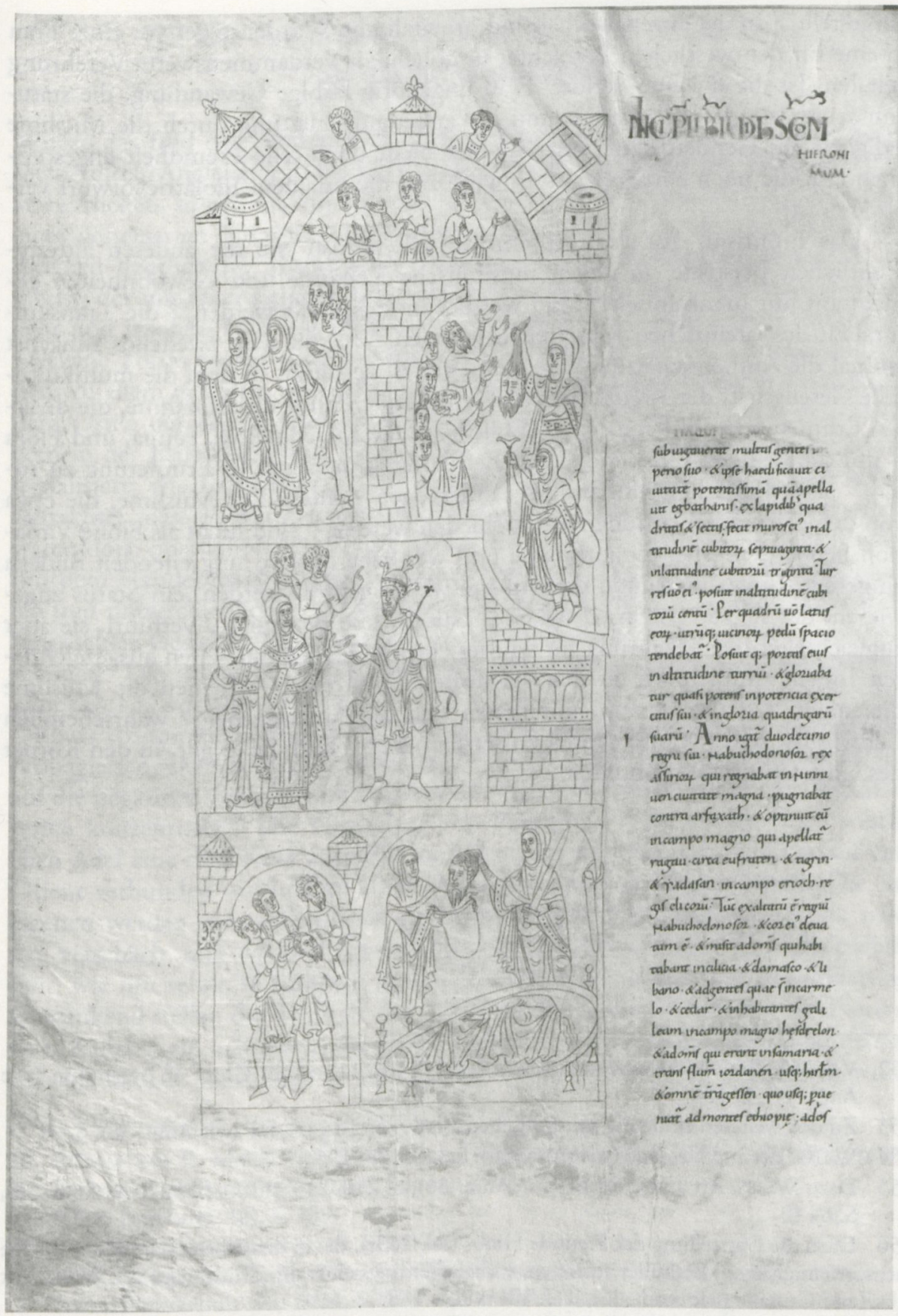

Abb. 6: Frontispiz zum Buch Judith. Roda Bibel, Spanisch, 11. Jahrhundert. Paris, Bibliothèque nationale de France: Ms. lat. 6, fol. 134v 
dargestellt. ${ }^{52}$ In der Arsenal-Bibel sind orientalische Würdenträger vor einer Frau in eine für den westlichen Betrachter unübliche, ja verdammenswerte Verehrung gefallen. Ist die für eine 'seriöse' Frau ungehörig farbige Gewandung, die statuenhaft erhöhte Repräsentation und die anbetende Haltung durch die Muslime lediglich ein Genderdiskurs oder ist hier nicht doch jene Fremdheit angesprochen, auf die noch einzugehen sein wird und die mit dem Idolatrievorwurf verbunden ist?

Das Frontispiz zur Judithgeschichte will ebenso wie die anderen alttestamentlichen Beispiele, in denen muslimische Kostüme und Gewohnheiten gemeinsam mit byzantinischen und westlichen dargestellt werden, ${ }^{53}$ die Transkulturalität des lateinischen Königreichs betonen. ${ }^{54}$ Damit wird zweifellos zunächst einmal die Authentizität des 'Heiligen Landes' verstärkt, in dem die multikulturelle Gesellschaft der Kreuzfahrer existiert. Freilich muss die Annahme, die diversen Ethnien seien differenziert - ähnlich wie etwa Architektur, Fauna, und Flora - um die heiligen Orte und deren Befreiung authentisch in Erinnerung zu rufen, ${ }^{55}$ einer kritischen Überprüfung unterzogen werden. Die Muslime, die etwa von den orientalischen Juden unterschieden werden, ${ }^{56}$ sind nicht als bloße Variation neben den sonst byzantinische und westliche Motive aufgreifenden Bildern eingefügt. Ihnen wird, und dies müsste weiter geprüft werden, ein Status zugewiesen, der sie zur Uneinsichtigkeit - Balaam, die Bethulier - verführt, sie aber zugleich eher der Bekehrung für würdig erachtet als die nur selten ausgezeichneten Juden. Ähnlich wie in den "Histoires d'Outremer» scheinen die Muslime diejenigen Akteure zu sein, mit denen sich die Auftraggeber - wahrscheinlich Ludwig IX. selbst - auseinander zu setzen haben ${ }^{57}$ und die es gilt, in den Körper des Christentums einzubinden.

52 Venedig, Biblioteca Marciana: Ms. gr. Z 17, fol. 111; Abb. Glory of Byzantium (wie Anm. 49), S. 186.

$53 \mathrm{Zu}$ den weiteren eindeutig muslimischen Verweisen BUCHTHAL (wie Anm. 36), S. 65.

54 WeIsS, Art and Crusade (wie Anm. 36), bes. S. 187-195.

55 Dazu Weiss, Art and Crusade (wie Anm. 36), S. 210-215 - BuCHTHAL (wie Anm. 36), S. $64 \mathrm{ff}$.

56 Dazu die Darstellung der Freunde Hiobs (fol. 269r), die in denselben Gewändern wie die orientalisierten Bethulier auftreten, wobei allerdings einer die Zizith trägt; vgl. WEISS, Art and Crusade (wie Anm. 36), Taf. VII und S. 188.

57 In der Amalekiterschlacht etwa sind die beiden Heere nicht voneinander unterschieden, sowohl Israeliten wie Amalekiter tragen mameluckische Rüstungen; vgl. WEISS, Art and Crusade (wie Anm. 36), Abb. 36 zu Robinson (wie Anm. 41), Taf. VI und VII. 


\section{Die Faszination des Fremden und Aneignung als das zugleich Eigene}

Wurde das Bild der Sarazenen in einigen Kreuzfahrerhandschriften dazu eingesetzt, durch Angleichung der beiden Parteien eine gemeinsame Ritterkultur zu verherrlichen, so nehmen im 14. und vor allem im 15. Jahrhundert die Zeugnisse eines gesteigerten Interesses an einer konkretisierenden Schilderung der Andersartigkeit deutlich zu. Mimetisches Darstellen ist ein Paradigmenwechsel in der Kunst, der mit einer sich verändernden Erfahrungswelt der Zeitgenossen einhergeht und insofern auch das Bild des Ostens einem veränderten Blick unterwirft. Der eine Topos weicht einer Vielfalt von Topoi. Bereits im 14. Jahrhundert werden vor allem in Italien unterschiedliche, nun auch rassisch definierte Fremde aus dem Osten dargestellt. Giottos präzise geschilderter Schwarzer unter den Peinigern in der Darstellung der Verspottung Christi in der Arena-Kapelle in Padua signalisiert, wie Rosamond E. Mack annimmt, auch für den zeitgenössischen Betrachter, dass es sich hier um einen aus Afrika stammenden Sklaven handelt, waren doch oberitalienische Städte bereits seit dem ausgehenden 12. Jahrhundert mit dem Handel muslimischer Afrikaner befasst. ${ }^{58}$ Zunehmend werden Mongolen und unterschiedliche Völkerstämme aus Asien konkretisiert und ihre charakteristischen Gesichtszüge, vor allem aber Details ihrer Gewandung, genau geschildert. Die genauere Beobachtung und Darstellung hängt auch mit dem im Lauf des 13., vor allem aber des 14. Jahrhunderts sich verschärfenden rassischen Diskurs $^{59}$ zusammen, der sich im Rahmen des vor allem durch die Bettelorden propagierten, zur Compassio anregenden Passionsrealismus in besonderer Weise auf die negativen Gestalten im Kontext der Passion Christi und der Heiligenmartyrien konzentriert. ${ }^{60}$ Die Niederlage von Nikopolis 1396 scheint beim westlichen Adel eine tiefe Krise ausgelöst zu haben, die mit einer neuen Sicht auf den Orient verbunden war und zu einer Differenzierung der Gegner führte: Die Sarazenen werden nun zunehmend zu Türken. ${ }^{61}$ Die Kreuzzugspropaganda wird neu verschärft, und das Bild der osmanischen Türken ist in einem Maße polarisiert, wie nur selten in früheren Zeiten. Zwischen Schrecken und Bewunderung schwankend stellen die Texte sie dar. 1397 verfasst Coluccio Salutati, der Florentiner Kanzler, ein Schreiben, das große Verbreitung fand und dessen Argumentation bis ins 16. Jahrhundert weiter tradiert werden sollte. ${ }^{62}$ Er schildert bewun-

58 Rosamond E. MaCK: Bazaar to Piazza. Islamic Trade and Italian Art, 1300-1699. Berkeley (Ca) / Los Angeles (Ca) / London 2002, S. 151-153.

59 HENG (wie Anm. 18), S. 139.

60 STRICKLAND (wie Anm. 14), S. 173-175.

61 NANCY BISAHA: Creating East and West. Renaissance Humanists and the Ottoman Turks. Philadelphia (Pa) 2004, S. 55 f. - JOYCE KUBISKI: Orientalizing Costume in Early Fifteenth-Century French Manuscript Painting (Cité des Dames Master, Limbourg Brothers, Boucicaut Master, Bedford Master). Gesta 40 (2001) S. 161-180, bes. 162 f. 
dernd die Kraft des türkischen Heeres, dessen Ausbildungsgänge und bringt vor allem die Schwäche des Westens mit dessen fehlender Entschiedenheit und Erziehung im Glauben zusammen. Die Christen, die nicht im Stande seien das Schisma zu beenden, werden den osmanischen Türken gegenübergestellt, deren Glaubenskraft - auch im Heiligen Krieg - und Geschlossenheit er seinen Zeitgenossen als Spiegel vorhält; eine Argumentation, die auch in der Türkenpropaganda des 16. Jahrhunderts noch zu finden sein wird. ${ }^{63}$ Vor diesem Hintergrund dürften auch die sehr präzisen Darstellungen osmanischer und östlicher Gestalten im Umkreis des französischen Hofes zu interpretieren sein. Eine weitere Aufwertung erfahren Türken in ihrer Identifizierung mit den Trojanern, sind sie doch damit an Anciennität den Römern überlegen, die sich ja ihrerseits auf die Trojaner zurückführen. ${ }^{64}$

\section{a) Orientalisierung und Ermahnung}

Die topische Tradierung und gleichzeitige Instrumentalisierung des Sarazenen eröffnet in einer Augustinus-Handschrift aus dem zweiten Jahrzehnt des 15. Jahrhunderts einen Subtext im zeitgenössischen Rahmen. ${ }^{65}$ Die Miniatur zum Exempel der Begegnung des Makedonenkönigs Alexander des Großen mit dem Piraten (Abb. 7) beschäftigt sich mit der richtigen Regentschaft. Ein Pirat nähert sich in seinem Schiff und fragt Alexander, was denn der Unterschied zwischen seinem und des Herrschers Verhalten sei. Er kämpfe mit seinem kleinen Schiff und werde Pirat genannt und Alexander mit einer Flotte und heiße Admiral. Der Maler, der für bedeutende Auftraggeber gearbeitet hat, ${ }^{66}$ überträgt das belehrende Exempel in eine übliche europäische Landschaft und kleidet den in seinem Boot sitzenden Piraten in eine abenteuerliche Rüstung, die eindeutig orientalisierend

63 BisAHA (wie Anm. 61), S. 161-166.

64 JAMES G. HARPER: Turks as Trojans; Trojans as Turks. Visual Imagery of the Trojan War and the Politics of Cultural Identity in Fifteenth-Century Europe. In: Postcolonial Approaches to the European Middle Ages. Translating Cultures. Hg. von ANANYA JAHANARA KABIR und DEANNE Williams. Cambridge 2005, S. 151-179 - BiSAHA (wie Anm. 61), S. 56.

65 Paris, BnF: Ms. fr. 20 und 21; dazu Ines Villela-Petit: Deux visions de la Cité de Dieu. Le Maître de Virgile et le Maître de Boèce. Art de l'enluminure 17 (2006) S. 2-19, S. 28-29 - SHARON DUNLAP SMITH: New Themes for the "City of God" around 1400. The Illustrations of Raoul de Presles' Translation. Scriptorium 36 (1982) S. 68-82. Zur Zuschreibung an den Boethius-Meister SHARON OfF DUNLAP SMITH: Illustrations of Raoul de Praelles' Translation of St. Augustine's "City of God" between 1375 and 1420. Diss. New York University 1974 (ProQuest Dissertations \& Theses Full Text, Publication Number AAT 48106), S. $146 \mathrm{ff}$.

66 Villela-Petit (wie Anm. 65), S. 19, gibt als Provenienz den Buchhändler Jean Bonhomme an, der die Handschriften 1488 von Jean Cueillette, Schatzmeister des Pierre de Beaujeu, Herzog von Bourbon gekauft haben will; zu weiteren Auftraggebern des Boethius-Meisters: Paris 1400. Les arts sous Charles VI. Ausstellungskatalog Musée du Louvre, Paris 2004. Paris 2004, Kat. 174, S. 283 f. 


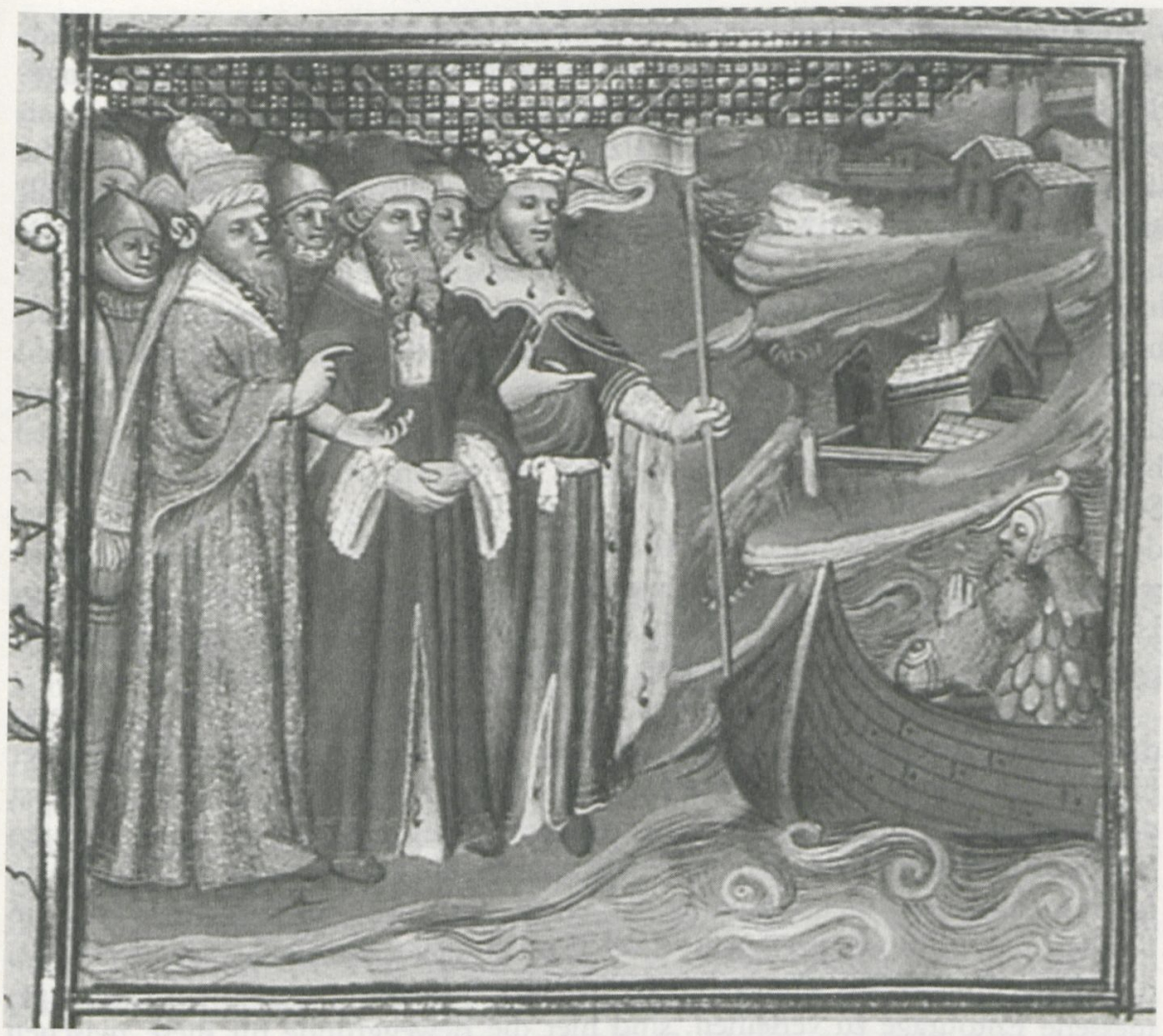

Abb. 7: Alexander und der Pirat. Augustinus, "Cité de Dieu», Paris: Boethius-Meister, um 1415. Paris, Bibliothèque nationale de France: Ms. fr. 20, fol. $111 \mathrm{v}$

gestaltet ist. Eine Beckenhaube, die allerdings einen Nasenschutz kennt, wie er bei türkischen und mameluckischen Helmen üblich ist ${ }^{67}$, wird kombiniert mit einem goldenen Kettenpanzer als Nackenschutz. Riesige goldene Achselrosetten, die in Lamellen auslaufen, gelten üblicherweise als imponierende Accessoires fremder Kämpfer. ${ }^{68}$ Ein Schuppenpanzer bedeckt den Körper. Nicht um eine konkrete Rüstung handelt es sich in dieser wohl um 1414 hergestellten Handschrift, sondern um eine Zusammenstellung unterschiedlicher Modelle, die andere Maler desselben Milieus durchaus sehr genau studiert und aufgegriffen haben. ${ }^{69}$ Der Makedonenkönig selbst, der mit dem Piraten debattiert, ist nun keineswegs der orientalische Potentat, den man erwarten würde, sondern ist

\section{RobInSON (wie Anm. 41), Tafel IX.}

68 So etwa Feirefiz im Heidelberger "Parzival», Heidelberg, UB: Cod. Pal. germ. 339, fol. 540v; URL: <http://digi.ub.uni-heidelberg.de/diglit/cpg339ii/0536> (17.2.09).

69 KUBISKI (wie Anm. 61), S. 165 f. 
vielmehr zu einem Zeitgenossen geworden, den man mit dem französischen König assoziiert. Die Lilienkrone auf dem Haupt, ist er in die von Charles V. eingeführte graue Robe gekleidet ${ }^{70}$, die er allerdings gegürtet trägt und die vorne einen modischen Schlitz besitzt, der das Pelzfutter vorblitzen lässt. Unter dieser Robe sieht man die engen Ärmel einer goldfarbenen Tunika, über die Schultern fällt ein bodenlanger, blauer Mantel, über dem ein breiter Zobelkragen liegt. In der Linken präsentiert er die Fahnenlanze. All dies sind Elemente der vertrauten herrscherlichen Darstellung eines französischen Königs. Seine Begleiter erst definieren den mit einem kurzen, zweigeteilten Bart gezeigten König als nicht westlichen Potentaten in einer 'fremden' Umgebung. Mit ihren konischen Hüten und der um den Kopf geschlungenen Binde, deren Enden weit über den Rücken hängen, sind beide Gestalten orientalisiert. Sie tragen zwar nahezu dieselben an Schauben gemahnenden Roben wie der König, diese sind aber, vor allem bei dem heftig Gestikulierenden, mit kostbaren Besätzen versehen, auf denen ebenso wie an den Binden eingewebte kufische Zeichen angedeutet werden. Alexanders Heer ist wiederum ein rein westliches, tragen doch die Soldaten die übliche zeitgenössische Beckenhaube mit der eng anschließenden Helmbrünne.

Die orientalisierten Pairs spielen im Kontext der Belehrung des Herrschers durch den Piraten eine wichtige Rolle, die ihnen auch in anderen Bildern der Handschrift zukommt. Sie sind die weisen Diskutanten, die diesem Exempel erst die entscheidende Deutung verleihen, so sind sie es auch, die in einer weiteren Miniatur über Gott debattieren. ${ }^{71}$ Nicht eine zufällige Mode oder das allgemeine Interesse an einer Orientalisierung der Mode, das sowohl in Italien wie auch im Norden seit 1400 anzutreffen ist ${ }^{72}$, auch nicht allein ein historisierender Wille, dem Makedonenkönig durch die orientalisierten Begleiter wenigstens eine angemessene Entourage zu verleihen, sind hier die Beweggründe für diese zwischen östlichen und westlichen Traditionen vermittelnden Figuren. Zwar ist die Beobachtung Ivan Davidson Kalmars richtig, dass eine einzige 'orientalisierte' Gestalt in einer Gruppe diese gesamthaft in den Orient versetzt. ${ }^{73}$ Damit wird auch der Makedonenkönig in gewisser Weise orientalisiert. Nun ist freilich die Hauptfigur als westlicher, genauer als französischer König dargestellt und das Exempel somit als Belehrung durch einen hybridisierten Fremden, einen Piraten, an den Regenten in der Zeit interpretiert. Ihm zur Seite stehen jene Weisen aus dem Orient, die auch das Gespräch über die Gottesfrage führen, und sie sind denn auch die Akteure, die redend und hörend das Thema erörtern. Der 'Orientalis-

70 STERLING (wie Anm. 35), Abb. 108.

71 Paris, BnF: Ms. fr. 20, fol. 212, URL: <http://visualiseur.bnf.fr/CadresFenetre? $\mathrm{O}=$ COMP-1\&I=17\&M=imageseule $>$ (17.2.09) - Frau Anja Eisenbeiß M. A. sei für Anregungen zu dieser Handschrift gedankt.

72 Dazu ReIner Haussherr: Convenevolezza. Historische Angemessenheit in der Darstellung von Kostüm und Schauplatz seit der Spätantike bis ins 16. Jahrhundert. Wiesbaden 1984 (Akademie der Wissenschaften und der Literatur Mainz, Abhandlungen der Geistes- und Sozialwissenschaftlichen Klasse. Band 1984,4), S. 13 f.

73 Kalmar (wie Anm. 24), S. 29-31. 
mus' in dieser Handschrift dürfte somit weniger auf eine zeitliche und örtliche Situierung des Ereignisses hinzielen, als vielmehr eine autoritative Verstärkung der Belehrung durch die orientalischen Philosophen anstreben. Als Subtext dürfte damit auch ein politischer Aufruf zur Einbindung 'fremder' Denker in die Reihen der Krone verbunden sein. Die Handschrift muss in den bibliophilen Kreisen des Hofes entstanden sein und ist um 1415 in einer Situation geschaffen worden, in der das abendländische Schisma, der Hundertjährige Krieg, die Armagnakenkriege und der geschwächte König Charles VI. die Krone und den Herzog von Berry in eine labile Lage gebracht haben. ${ }^{74}$ Vor diesem Hintergrund ist das Exempel mit dem Piraten, erörtert von den Weisen des Orients, eine deutliche Ermahnung, die richtige Regentschaft zu bedenken.

\section{b) Orientalisierung als Identifikationsangebot}

Eine wesentlich komplexere Form der Orientalisierung, die zugleich mit einer Identifizierung gerade mit dem Orient verbunden ist, kann an der eigenartig hybriden Augustusgestalt in den "Très Riches Heures» des Duc de Berry (Abb. 8) dekonstruiert werden. Die Vision des Kaisers Augustus, der gemeinsam mit der tiburtinischen Sibylle Maria mit dem Kind in einem Strahlenkranz sieht, ${ }^{75}$ ist eines der Themen, das vom Duc de Berry und dessen Umkreis nicht nur geschätzt, sondern entschieden gefördert wurde. ${ }^{76}$ Der römische Kaiser Augustus, bereits bei Petrarca als Inbegriff des heroischen Vereinigers beschrieben, ${ }^{77}$ wird in dieser Darstellung zum Orientalen. Er trägt einen langen weißen Bart und einen hohen Hut, dessen vertikale Segmente mit goldenen Kanten und Perlschnüren geschmückt sind und auf dessen Spitze ein roter Stein sitzt. Die mit Pelz verbrämte, gespaltene Krempe ist hoch geschlagen. Es dürfte sich um eine Variante jenes Hutes handeln, mit dem Pisanello Johannes VII. Paläologus darstellen sollte und der in der westlichen bildenden Kunst eine wiederholte Aufnahme fand. ${ }^{78}$ Mit dieser Kopfbedeckung ist wohl eine dem Kaiser der Paläologenzeit vorbehaltene Kopfbedeckung gemeint, ${ }^{79}$ die - wie Joyce Kubiski nachweist - freilich ihrerseits von asiatischen Kronenhüten beeinflusst ist. ${ }^{80}$ Insbesondere die steile Form

74 Françoise Autrand: Jean de Berry. L'art et le pouvoir. Paris 2000, S. 197-243.

75 Artikel „Augustus“. In: Lexikon der christlichen Ikonographie 1 (1968), Sp. 225-227 GERTRUD SCHILLER: Ikonographie der christlichen Kunst. Bd. 1. Gütersloh ${ }^{3} 1981$, S. 92 f. und Bd. 4.2. Gütersloh 1980, S. 215 f. - Eva SEBALD: Augustus und Sibylle - Ara Coeli. In: Marienlexikon 1 (1988), S. 300.

76 Millard MEISS: French Painting in the Time of Jean de Berry. The Late Fourteenth Century and the Patronage of the Duke. 2 Bde. London / New York 1967 (National Gallery of Art, Kress Foundation Studies in the History of European Art. 2), Bd. 1, S. 233-235.

77 BISAHA (wie Anm. 61), S. 51-53.

78 KUBISKI (wie Anm. 61), bes. Abb. 2.

79 PARANI (wie Anm. 42), S. 70.

80 KUBISKI (wie Anm. 61), S. 163. 


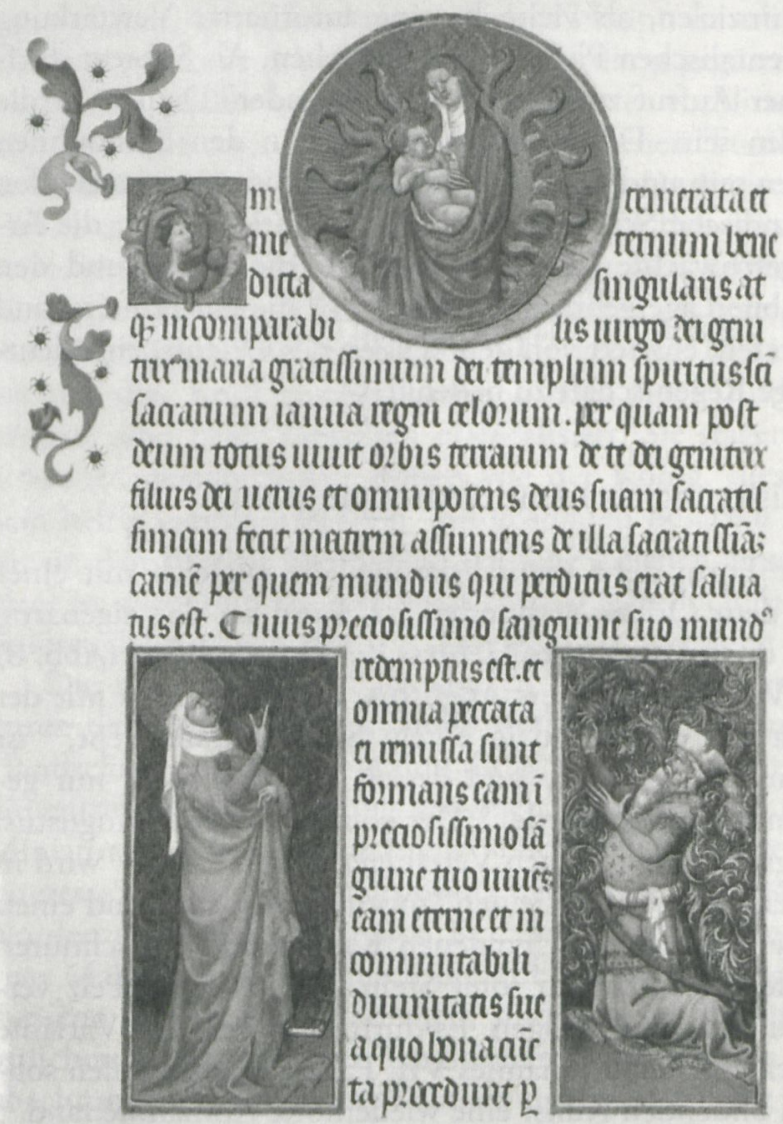

Abb. 8: Vision des Kaisers Augustus. "Très Riches Heures» des Duc de Berry, Gebrüder Limbourg, 1416. Chantilly, Musée Condé: Ms. 65, fol. 22 
und die Verzierung der 'Melonenschnitze' durch Goldauflagen und Perlschnüre entsprechen persischen wie auch mongolischen und türkischen Modellen. ${ }^{81}$ Über seinem mit goldenen Tiraz durchwirkten Gewand trägt Augustus ein gelbes Lederwams, das als Schutz in dieser kurzen Form nicht dem mittelalterlichen Lentner entspricht, sondern eher dem orientalischen Brustschutz. ${ }^{82}$ Das Wams ist mit goldenen heraldischen Zeichen besetzt, die eine eigenartige Unbestimmtheit besitzen und sehr verwandt, nun allerdings auf einer über einem Tappert getragenen Brigantine, bei David erscheinen in den beiden Miniaturen zu dem großen Dankgebet (Ps 99/100) und der Vision des auferstehenden Gottessohnes (Ps 62/63). ${ }^{83}$ Die Zeichen changieren zwischen Kreuzen, Blumen und 'werdenden' Fleur de Lys. Um die Hüfte trägt Augustus ein geknotetes, mit Goldstreifen durchwirktes Tuch, das wiederum eher in ein allgemein orientalisierendes Repertoire gehört. An einer goldenen Kette hängt an zwei längeren goldenen Riemen sein sehr präzise dargestelltes Krummschwert in der Scheide. ${ }^{84}$ Sowohl die Art der Befestigung wie auch die Verzierung der Schwertscheide, der geschwungene Knauf und der Schmuck der Parierstange verweisen auf orientalische, wohl mameluckische Waffen. ${ }^{85}$

Die Perfektion, mit der gerade die Brüder Limbourg, aber in noch viel stärkerem Maß der Cité des Dames Meister unterschiedliche Realien, Kostüme und auch Gesichter aus dem Nahen und Fernen Osten in den ersten Jahren des 15. Jahrhunderts darstellen, lässt auf eine breite Kenntnis ganz unterschiedlicher Kostümgewohnheiten östlicher Herkunft schließen. Die übliche Erklärung ist der Besuch Manuels II. Paläologus in den Jahren zwischen 1399 und 1402 in London und Paris, der mit seinem Gefolge große Aufmerksamkeit erregte. ${ }^{86}$ Zudem scheinen mongolische, türkische und persische Handschriften zirkuliert zu haben. ${ }^{87}$ Die vielen aus dem Nahen und Fernen Osten importierten Waren,

81 KUBISKI (wie Anm. 61), Abb. 5a.

$82 \mathrm{Zu}$ dem kurzen Wams ROBINSON (wie Anm. 41), Taf. VI. - Denselben Brustschutz trägt der älteste der Heiligen Drei Könige auf fol. 51v, der auch in den anderen Details der Gewandung weitgehend mit Augustus übereinstimmt; dazu KALMAR (wie Anm. 24), S. $3 \mathrm{f}$.

83 Chantilly, Musée Condé: Ms. 65, fol. 39v; Abb. Les "Très Riches Heures" du Duc de Berry, Faksimile des Originals, das sich unter der Nr. 65 im Musée Condé in Chantilly befindet. Luzern 1984.

84 Abb. DAVID C. NiCOLlE: Arms and Armour in the Crusading Era, 1050-1350. 2 Bde. White Plains (NY) 1988, Bd. 2, S. 694, Abb. 328 und S. 696, Abb. 334 AJ.

85 Turks. A Journey of a Thousand Years, 600-1600. Ausstellungskatalog Royal Academy of Arts, London 2005. Hg. von DAVID J. ROXBURGH. London 2005, Abb. 158 und 249 zum Schwert, zum Schwertgehänge Abb. 162.

86 JOHN W. BARKER: Manuel II Palaeologus (1391-1425). A Study in Late Byzantine Statesmanship. New Brunswick (NJ) 1969, S. 167 ff. - KUBISKI (wie Anm. 61), S. 175 KALMAR (wie Anm. 24), S. 5 f.

87 KUBISKI (wie Anm. 61), S. 175 f. 
die als bewunderte Luxusgüter galten, dürften weitere Kenntnisse vermittelt haben. ${ }^{88}$

Freilich kann die veränderte Aufmerksamkeit und das Interesse für das Orientalische, das durchaus im 15. Jahrhundert modischen Charakter zu entwickeln scheint, Darstellungen wie diejenige des Augustus nicht erklären. Nicht allein die Freude am Fremdländischen kommt in solchen Montagen zum Ausdruck, wird doch an diesem Beispiel des Augustus deutlich, dass die historische Figur in ein komplexes Verweissystem eingespannt ist. Ein römischer Kaiser, der mit der Legende seiner Vision offenbar für den Auftraggeber von besonderer Bedeutung gewesen ist, kann doch der Duc de Berry als wichtigster Beförderer dieses Themas $^{89}$ angesehen werden, wird zu einem orientalischen Fürsten, in dem Oströmisch-Byzantinisches mit Mameluckischem, Türkischem und Persischem verschmilzt. Zugleich schafft seine Gestalt innerhalb der Handschrift einen Bezug zu den Heiligen Drei Königen auf ihrer Reise zu dem Gottessohn, auf der sie ebenfalls einer Vision, dem Stern, folgen. ${ }^{90}$ Mit der Verwandtschaft zu David schließt sich ein typologischer Kreis, der die alttestamentliche Vision Davids über den die Geburt des Gottessohnes sehenden römischen Kaiser zu den Heiligen Drei Königen verfolgt, die ihn 'leibhaftig' berühren können. ${ }^{11}$ Die typologische Vernetzung ist, wie Margaret M. Manion am Beispiel der Psalmenillustrationen belegt, ein zentrales Anliegen des Programms dieser Handschrift. ${ }^{92}$ Ebenso wird das für ein Stundenbuch unübliche Verständnis des Liturgischen ${ }^{93}$ auch in dieser Darstellung zum Ausdruck gebracht. Augustus erlebt nicht nur die Vision, sondern empfängt diese in einer liturgischen Situation, schwenkt er doch das Weihrauchfass. ${ }^{94}$

Die Interpretationsangebote der Augustusgestalt sind vielfältig. Der typologische Verweis wird mit einem liturgischen verbunden. Insbesondere in der Einbindung unterschiedlicher zeitlicher und ethnischer Bezüge wird ein Anspruch deutlich, der in manchen Psalmendarstellungen noch klarer formuliert wird. Wenn zu Psalm 8 (Abb. 9), in dem David Gottes Schöpfung besingt, unter dem über Wolken erscheinenden Christus-Logos die Menschen, denen Gott sich zuwendet, als eine Gruppe schwarzer hochrangiger Mamelucken gezeigt wer-

88 MACK (wie Anm. 58), S. 20-24.

89 Millard MeIss: French Painting in the Time of Jean de Berry. The Limbourgs and their Contemporaries. 2 Bde. London 1974 (The Franklin Jasper Walls Lectures. Band 2), Bd. 1, S. 64 .

90 Chantilly, Musée Condé: Ms. 65, fol. 51v; Abb. Faksimile (wie Anm. 83).

91 Chantilly, Musée Condé: Ms. 65, fol. 52r; Abb. Faksimile (wie Anm. 83).

92 Margaret M. Manion: Psalter Illustration in the Très Riches Heures of Jean de Berry. Gesta 34 (1995) S. 147-161, bes. 151.

93 MANION (wie Anm. 92), S. 148.

94 In keiner der anderen Darstellungen ist Augustus vergleichbar orientalisiert und aktiv, vgl. dazu MEISs, Limbourgs (wie Anm. 89), Abb. 399 und ebenfalls von den Gebrüdern Limbourg, Abb. 397. 


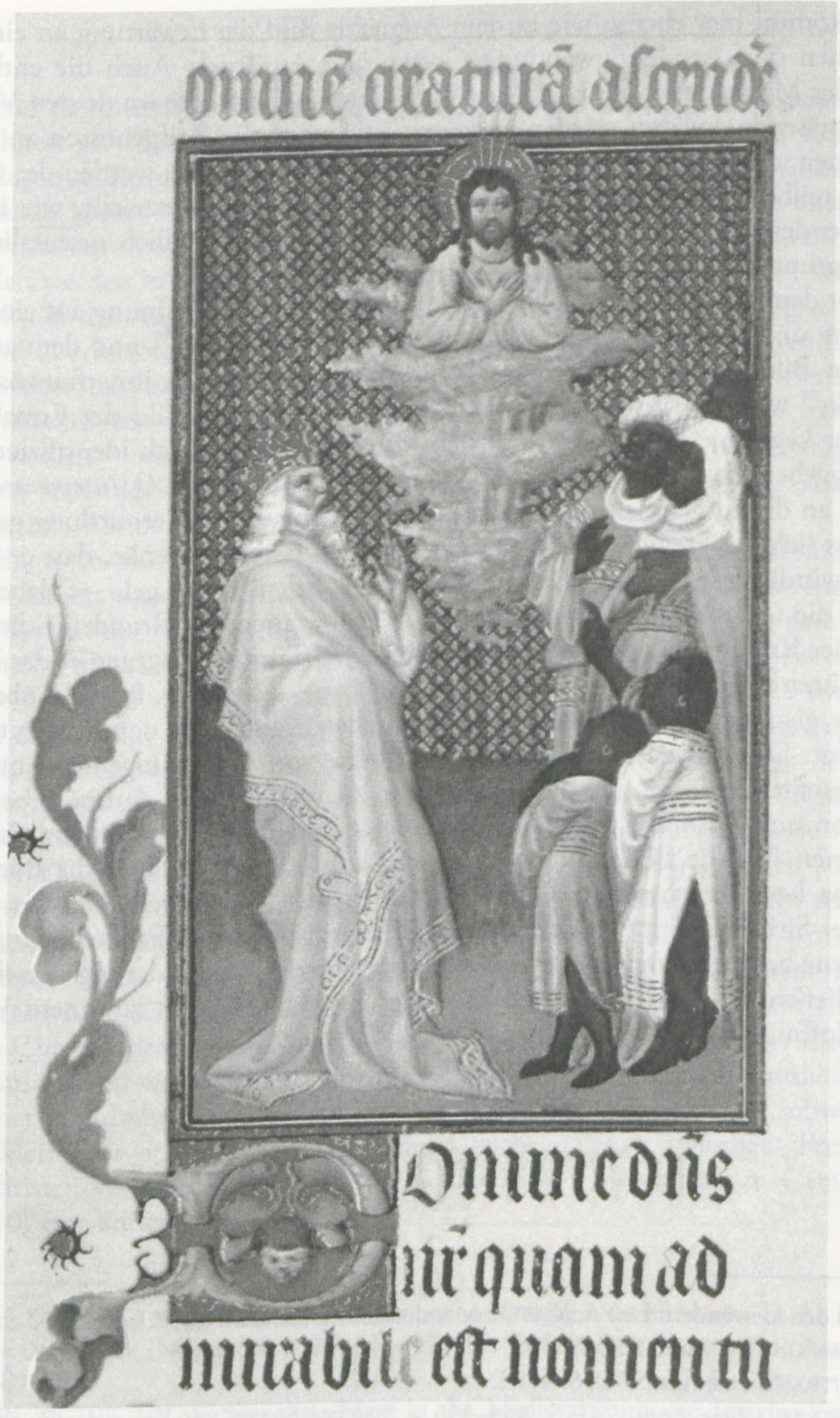

Abb. 9: Davids Lobpreis (Ps 8). "Très Riches Heures» des Duc de Berry, Gebrüder Limbourg, 1416. Chantilly, Musée Condé: Ms. 65, fol. 27v 
den ${ }^{95}$, kommt hier ebenso wie in dem Augustus-Bild die Erwartung an eine alle Menschen umfassende Zuwendung Gottes zum Ausdruck. Auch die entblößte Brust der Mameluckin, die von dem Wort der Säuglinge, die im dritten Vers als Gott Preisende erwähnt werden, angeregt ist ${ }^{96}$, wird die Zeitgenossen an Maria gemahnen, die ihre Brust als Fürbittende weist. Die Fremden werden der Gnade Gottes teilhaftig oder werden mindestens durch das Wort erreicht, wie im anschließenden Bild (fol. 28), in dem die Apostel unterschiedlich orientalisierten Völkergruppen predigen.

Vor dem Hintergrund des Schwankens zwischen der Hoffnung auf eine Versöhnung und Beilegung des Schismas im Konstanzer Konzil ${ }^{97}$ und dem auch in anderen Bildern der "Très Riches Heures» angesprochenen innerfranzösischen Konflikt $^{98}$ wird die Vision des Kaisers Augustus zu einem Bild der Ermahnung und der Legitimierung für den mit dem römischen Kaiser sich identifizierenden Auftraggeber, den Duc de Berry ${ }^{99}$. Im zugehörigen Gebet $O$ Intemerata wird Maria, an die zu glauben die göttliche Gnade sichere, um Vermittlung gebeten, auf dass sich der Heilige Geist so in das Herz des Betenden senke, dass er Gottes Liebe würdig werde. Um Glaubensstärke und Erleuchtung geht es dabei, und gerade die Glaubensstärke war in Salutatis Propaganda der Grund für die Betonung der Kraft der für den Glauben kämpfenden Türken, wogegen er das Fehlen des wahren Glaubens als Ursache für das Schisma ausmachte. Im Bild überblendet der glaubensstarke Türke den oströmischen Kaiser, der damit orientalisiert erscheint. Die Darstellung dürfte in jene Reihe von Aneignungen 'orientalisierter' Gestalten gehören, die dem sich damit identifizierenden Auftraggeber Legitimation und Autorität verleihen sollen, seine Intentionen zu verdeutlichen, im konkreten Fall die Befriedung der innerfranzösischen Krise und die durch das Schisma heraufbeschworene Glaubenskrise. In der Zusammenschau der Zeiten und der Sicherheit im Glauben an eine Welt umspannende Existenz Gottes, die auch jene berühren und erleuchten wird, die sich der Missionierung derzeit noch verschließen, ist Augustus gerade in seiner hybriden Gestalt zu einem Vorbild und Hoffnungsträger geworden.

$95 \mathrm{Zu}$ den Gewändern LeO A. MAYER: Mamluk Costume. Genf 1952, bes. S. 59.

96 MANION (wie Anm. 92), S. 150.

97 Autrand (wie Anm. 74), S. 418 f.

98 Autrand (wie Anm. 74), S. 444-446 - Simona SlaniČKa: Krieg der Zeichen. Die visuelle Politik Johanns ohne Furcht und der armagnakisch-burgundische Bürgerkrieg. Göttingen 2002 (Veröffentlichungen des Max-Planck-Instituts für Geschichte. Band 182), S. 267-279.

99 MEISS, Limbourgs (wie Anm. 89), S. 64. 


\section{Der Andere als das Böse}

Während die orientalisierenden Motive in der Gestalt des Augustus dessen Vorbildlichkeit als Identifikationsgestalt verstärken, werden in anderen Beispielen eher negative Kombinationen gewählt. Die vor allem von Joyce Kubiski beobachteten absurden Montagen orientalisierender Elemente schaffen eine Form konkretisierten Andersseins, das neue Züge erhalten hat. Die Gewänder der Fremden werden in ihrer Übertriebenheit, ihrem unbekannten Luxus - wie sogar am Beispiel des Augustus -, in ihrer unvertrauten Zusammenstellung zu einem kulturell überformten und neu montierten Hybridkörper, der in älteren Darstellungen eher in Form physischer Monstrositäten somatisiert wird. Bereits um einen reflektierten Umgang mit dem Akt der Dämonisierung von Sarazenen dürfte es sich - wie schon erwähnt - bei den dämonische Masken tragenden Gegnern Karls des Großen vor Córdoba handeln, wie sie in der für Charles V. hergestellten "Grandes Chroniques de France» gezeigt werden.

Geht es in solchen Montagen zunächst um ein Darstellen 'be-fremdlicher' Unvertrautheit durch Maßlosigkeit und Übertreibung, die in dem streng regulierten System der Kleiderordnungen durchaus lächerlich wirkt, so ist damit dennoch nicht unbedingt eine generell negative Wertung verbunden. Bereits seit dem Frühmittelalter, aber erstaunlich selten werden Muslime zu den Anderen, auf die das absolut Böse projiziert wird. Wenige Bilder des Propheten begleiten die weit verbreitete schriftliche Propaganda, die ihn als verwerflichen Sektierer und bösen Verführer zeiht. ${ }^{100}$ Am breitesten wird die anti-islamische Propaganda im Spätmittelalter im Rahmen des Idolatrievorwurfs ausgelebt. Muslime werden in dieser Darstellung zu den Heiden par excellence, die oft mit den Römern gleichgesetzt werden und mit diesen die Anbetung von Götzenbildern teilen. ${ }^{101}$ Nicht selten werden denn die Idole selbst zu Mohammeds, und die Vorstellung, der Prophet habe von sich ein Bildnis herstellen lassen und diesem mittels schwarzer Magie dämonische Kräfte eingeflößt, kommt etwa in der sehr einflussreichen Chronik des Pseudo-Turpin vor. ${ }^{102}$ Dass Idole Herrschaft über die Menschen ausüben ist eine Formel, die zum Inbegriff der Besessenheit wurde. In der Londoner Handschrift mit den unterschiedlichen Reiseberichten ist, obwohl in den Bildern, wie schon beobachtet, eine Gleichrangigkeit zwischen 'Fremden' und Christen verbildlicht wird, die Botschaft in der Illustration zu Campision (Abb. 10) eine andere. ${ }^{103}$

100 Dazu Michael Uebel: Ecstatic Transformation. On the Uses of Alterity in the Middle Ages. New York (NY) 2005, S. 25-36. - Zu den Bildern STRICKLAND (wie Anm. 14), S. 189-192.

101 JOHN VICTOR TOLAN: Saracens. Islam in the Medieval European Imagination. New York (NY) 2002, S. 126-134.

102 Dazu TOlan, Saracens (wie Anm. 101), S. 133.

103 Marco Polo: Das Buch der Wunder. Aus: "Le Livre des Merveilles du Monde», Ms. fr. 2810 der Bibliothèque nationale de France, Paris. Übersetzung der Handschrift MARIE- 


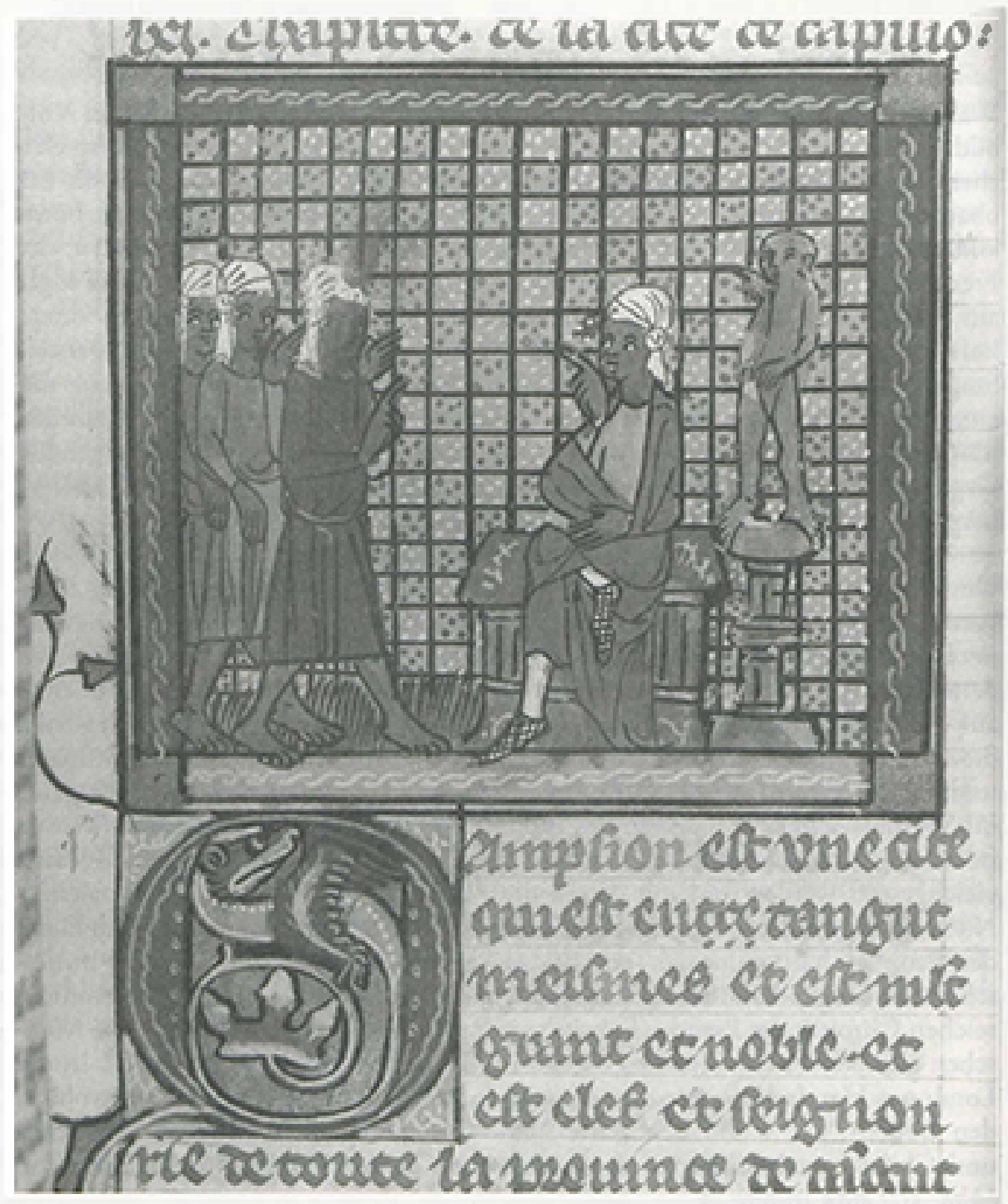

Abb. 10: Die Leute von Campision vor dem Vertreter des Großkhan. Reisehandschrift, Paris, nach 1333. London, The British Library: Royal 19 D I, fol. 76

Der Vertreter des Großkhans, der in westlicher Weise thronend mit übergeschlagenen Beinen und zeitgenössischen durchbrochenen Schuhen dargestellt ist, weist mit einem Zeigegestus auf die mit ihm redenden Sarazenen. Hinter dem

HÉLÈnE TESNIÈRE, mit Beiträgen von FranÇOIS AVRIL und MARIE-THÉRÈSE GOUSSET. München 1999, S. 63: Campicion als Kan-chou identifiziert. 
Herrscher steht auf einem goldenen Säulenpodest eine goldene nackte Statue, ein Idol, das den Herrschergestus vorgebend als die eigentliche Macht dargestellt wird. Gleichsam hinter dem Rücken des Herrschenden werden er und seine Untertanen als diejenigen denunziert, die unter der Gewalt eines Idols stehen. Dass die Untertanen mit nackten Füßen vor den Herrscher treten, ist wohl ebenfalls eher als sublime Inferiorisierung denn als besondere Frömmigkeit zu deuten, treten doch nur noch in zwei anderen Illustrationen Kontrahenten barfüßig auf, nämlich die auf das Gnadenbrot des Großkhan angewiesenen Armen (fol. 95r) und die zu Wildmännern mutierten, in Fell gekleideten Tibeter (Abb. 11).

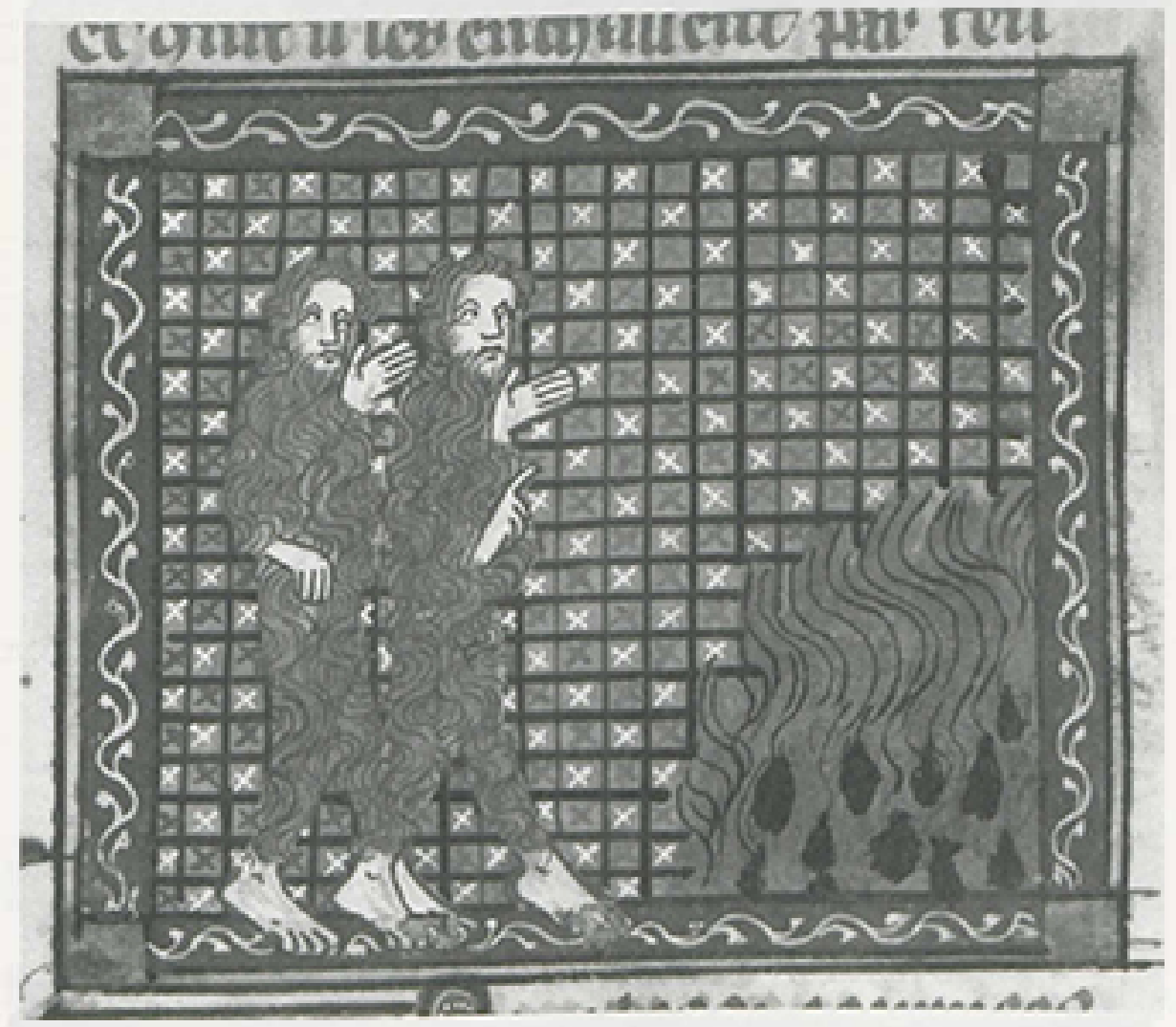

Abb. 11: Die Leute von Tibet in ihren Fellkleidern an ihren Feuern. Reisehandschrift, Paris nach 1333. London, The British Library: Royal 19 D I, fol. 98v

Einen breiten Pool an propagandistischen Bildern zur Denunziation aller Häretiker kennen die "Bible moralisée»-Handschriften des 13. Jahrhunderts. Dass in diesen Exemplaren im Bild nicht zwischen Juden, Römern und Muslimen unterschieden wird, kann nicht nur als Bildkonvention erklärt werden, sondern hängt entscheidend mit der Intention der Handschriften zusammen, die in einem zuvor 


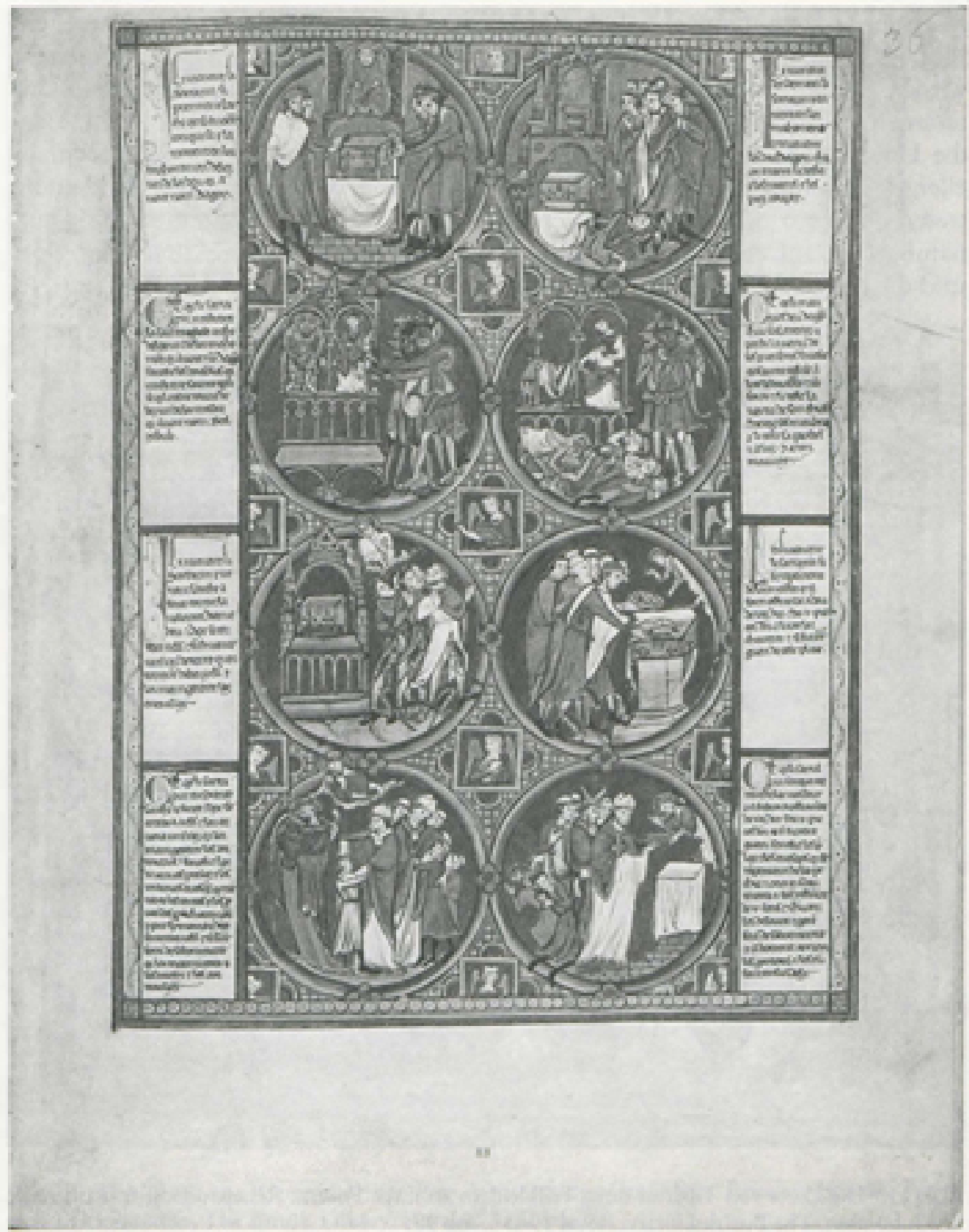

Abb. 12: Szenen aus 1 Könige 5, 2; 3-4; 6 und 6,5. "Bible moralisée», Paris, 1220-1226. Wien, Österreichische Nationalbibliothek: Cod 2554, fol. 36 


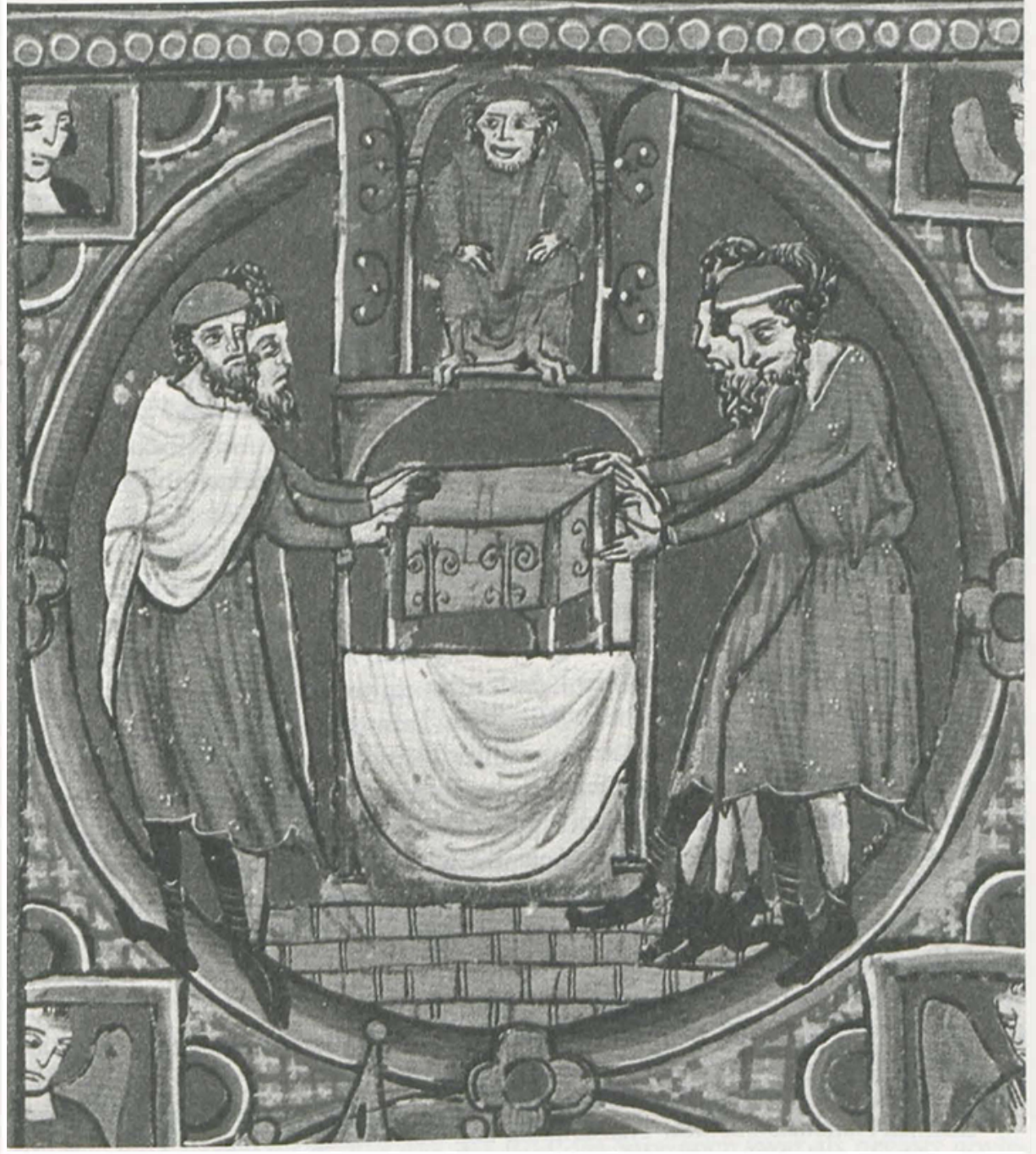

Detail aus Abb. 12: Die Bundeslade im Tempel des Dagon

nicht gekannten $\mathrm{Ma}$ einen Propagandafeldzug gegen jegliche Form der Häresie betrieben. ${ }^{104} \mathrm{Im}$ Bild werden besonders Juden dämonisiert, die gleichzeitig mit anti-semitischen Verfügungen ausgegrenzt werden. ${ }^{105}$ Juden und jegliche Art von Häretiker, Heiden, Nichtgläubige sowie Pervertierte und Tugendlose werden sowohl im zeitgenössischen Schriftum als auch in den Bildern unmittelbar aus-

104 SARA LIPTON: Images of Intolerance. The Representation of Jews and Judaism in the Bible moralisée. Berkeley (Ca) / Los Angeles (Ca) / London 1999, S. 49 f. und 82 ff.

105 LIPTON (wie Anm. 104), S. 6. 
tauschbar. ${ }^{106}$ Das Thema der Idolatrie wird geradezu zu einem Paradigma, das diese Gruppen gemeinsam charakterisieren soll. ${ }^{107}$ Die behauptete Verbindung zwischen Idolatrie und Habgier ist der Syllogismus, der diese Gruppen zusammenschweißt und auch visualisiert wird. In der französischen Version der "Bible moralisée», Codex 2554 der Österreichischen Nationalbibliothek in Wien, die wohl zwischen 1220 und 1226 für Louis VIII. entstanden sein dürfte, ${ }^{108}$ sind die negativen Gestalten in besonderer Drastik ins Bild gesetzt. Die Schänder der Bundeslade (Abb. 12), die Philister, werden im Text ${ }^{109} \mathrm{zu}$ Sarazenen und ihr Tempel zur mahommerie, zum Sitz des Islamischen und mithin Heidnischen schlechthin. ${ }^{110} \mathrm{Im}$ ersten Medaillon werden die sarrazin gezeigt, wie sie die geraubte Bundeslade unter den Tabernakel eines ihrer Götter, Dagon, stellen. Sie sind ununterscheidbar von den Juden in anderen Darstellungen und fallen ebenso wie die Israeliten in anderen Bildern durch ihren extremen Habitus auf. ${ }^{111} \mathrm{Im}$ Medaillon darunter wird der Vorgang erklärt: Der zum Belzebub gewordene Dagon befindet sich in einem Tabernakel neben Maria als Bild für die Kirche. Die sarrazin, die diese frevelhafte Zusammenstellung angezettelt haben, sind im Bild nun zu teuflischen Wesen geworden. ${ }^{112}$ Das Bild der sarrazin wird mit dem Götzendiener und dem Bösen schlechthin in eins gesetzt, das die Kirche bedroht.

Das Konstrukt des Sarazenen ist nicht nur mit Kampfeskraft, unendlichem Reichtum und Disziplin verbunden, sondern es eignet sich mithin auch dazu, in ihm das Böse schlechthin zu sehen. Wie Silke Tammen zu zeigen vermochte, ist denn nicht nur die Idolatrie als Inbegriff der Häresie in dieser Seite visualisiert, sondern die Götzenverehrung wird mit der Perversion der Homosexualität verknüpft. ${ }^{113}$ In der dritten Medaillonreihe werden die hartnäckig an der Schändung der Bundeslade festhaltenden Sarazenen von Gott bestraft, indem sie von Ratten überfallen werden. In der Moralisatio wird erklärt: „Dass die Sarraceni die Lade

106 LIPTON (wie Anm. 104), S. 83 ff.

107 LIPTON (wie Anm. 104), S. 40-42.

108 JOHN LOWDEN: The Making of the Bibles Moralisées. 2 Bde. University Park (Pa) 2000, Bd. 1, S. 90-94.

109 Dazu HanS-WaLter STORK: Bible moralisée. Codex Vindobonensis 2554 der Österreichischen Nationalbibliothek. Transkription und Übersetzung. St. Ingbert 1988 (Saarbrücker Hochschulschriften. Band 9: Kunstgeschichte), S. 104 f.: 88A-88D.

110 FRANÇOIS BERRIOT: Spiritualités, hétérodoxies et imaginaires. Études sur le Moyen Âge et la Renaissance. Saint-Étienne 1994, S. 148, wird der Begriff mahommerie mit Barbastro, der maurischen Festung, gleichgesetzt.

111 Vgl. etwa fol. 30, Abb. Bible moralisée. Faksimile-Ausgabe im Originalformat des Codex Vindobonensis 2554 der Österreichischen Nationalbibliothek. Graz 1973 (Codices selecti. Band 40).

112 Der Text der Moralisatio lautet: „Daß die Sarraceni die heilige Lade bei einem ihrer Götter, der den Namen Dagon hat, setzen, bedeutet die Teufel, die die heilige Kirche, die sie geraubt haben, zu einem ihrer Götter, der den Namen Beelzebub hat." Zitiert nach STORK (wie Anm. 109), S. 105: 88a.

113 Silke Tammen: Das Bild der Sodomie in der Bible Moralisée. Sexuelle und ideologische Perversionen. Frauen Kunst Wissenschaft 21 (1996) S. 30-48. 
mit Gewalt behalten und Gott ihnen zürnt und ihnen Ratten schickt, die ihnen die Eingeweide auffressen: das bedeutet die schlechten Bischöfe, die das Geld und den Zehnten durch Kauf und Simonie erhalten, und Gott zürnt ihnen und sie sind geschlagen mit Sodomie, die ihnen die Adern und die Eingeweide auffrisst" ${ }^{114}$ Beide Kontrahenten, die mit Sodomie geschlagenen Geistlichen, die im untersten Medaillon links gezeigt werden, wie sie sich mit Knaben abgeben und die Ecclesia verstoßen, und sogar die Sarazenen sind 'heil-bar'. Sie müssen bereuen und die Sarazenen in einer Art Analogiegeschenk fünf goldene Ratten und fünf Goldstücke dem wahren Gott opfern. Daraufhin fallen die Ratten von ihnen ab.

Eine Reihe ineinander verflochtener Vorurteilsthemen ist in dieser Seite angesprochen. Sodomie wird der Habgier zugerechnet, die durch eine Zuwendung zu Gott geheilt werden kann. Es ist die Kirche, die letztlich siegt - im zweitobersten Medaillon rechts -, indem die Marienstatue, die bereits schon im zweiten Medaillon links neben dem Gott Dagon zu sehen ist, diesen in der Darstellung daneben zerstört hat und die dämonischen Sarazenen ihr Idol nur noch an Haupt und Gliedern zerschmettert wieder finden. Als vom Teufel besetzte und dem Teufel folgende Häretiker sind die Sarazenen verstanden, die allerdings durch die Zuwendung zum richtigen Gott gerettet werden können. Häresie wird mit der Bilderverehrung gleichgesetzt und 'Fleischesverirrung' zu einer Folge der Häresie erklärt. ${ }^{115}$ Dies trifft auch auf die von der Sodomie befallenen Geistlichen zu, die mit den von den Ratten befallenen Sarazenen gleichgesetzt werden, ist doch ihr Impetus Habgier, die sie zu simonistischem Gebaren innerhalb der Kirche verführt. Die anti Jüdischen und Islam feindlichen Darstellungen der "Bible moralisée», die aufgehen in einem Vorstellungskomplex, der sich gegen die Feinde der Kirche insgesamt richtet, sind von der Intention der Handschriften geprägt, den Kampf gegen jegliche Häretiker zu propagieren und zu legitimieren. ${ }^{116}$

\section{Ausblick}

In den «Bibles moralisées» hat sich das Bild der Sarazenen zwar als negativ erwiesen, ist aber aufgegangen in dem großen Imaginaire der die Kirche bedrohenden Häretiker. Nicht einmal die üblichen Formeln sind dazu verwendet worden, eine Differenzierung der unterschiedlichen Gruppierungen zu erreichen. Der Text erst benennt die Figuren und konkretisiert die dämonischen und teuflischen Wesen als Muslime, was freilich ins Bild nicht aufgenommen wird. In den übrigen Beispielen hat sich die Darstellung des Sarazenen auf wenige Zeichen beschränkt. In der Regel konzentriert sich die Charakterisierung auf die Kopfbede-

114 Zitiert nach STORK (wie Anm. 109), S. 105: 88c.

115 Dazu auch LiPTON (wie Anm. 104), S. 105 ff.

116 LIPTON (wie Anm. 104), S. 132 f. 
ckung, einen Turban oder eine um den Kopf geschlungene Binde. Die Illustrationen der Londoner Reisehandschrift, in der alle Nichtchristen aus dem Nahen und Fernen Osten mit einer einzigen Formel - schwarzes Gesicht und um den Kopf geschlungenes Tuch - gezeigt werden, ändern je nach Kontext ihre Wertung. Ja, dieselbe Gestalt, nämlich der Khan, kann sogar in einem besonderen Zusammenhang, einer nach westlichem Muster verlaufenden Krönungsszene, durchaus weiß erscheinen und damit somatisch der Bedeutung des Rituals entsprechend sich verwandeln. ${ }^{117}$ Genau dies lässt sich auch in der "Bible moralisée» beobachten, in der die schließlich durch die Zuwendung zu Gott geretteten Sarazenen aus einem dämonischen Haufen ungeordneter Gestalten mit ausfahrenden Bewegungen zu gesittet ihr Opfer Darbringenden werden.

Muslime werden zwar wahrgenommen, gehen aber in den Bildern meist in der Gruppe der Sarazenen unter, unter die alle Nichtchristen subsumiert werden. Eine Wertung ist damit zunächst nicht verbunden, sondern diese wird durch den jeweiligen Kontext zum Ausdruck gebracht, so dass dieselbe Gruppe innerhalb einer Handschrift oder eines Zyklus durchaus unterschiedliche Bedeutungen gewinnen kann. Das zunehmende Interesse an einer Differenzierung der Gruppen und Akteure im 14. und vor allem im 15. Jahrhundert geht mit einer genaueren Beschreibung von Details sowohl der Kostüme als auch der Physiognomien einher. Ungewöhnliche Gewandkombinationen ebenso wie eigenartige körperliche Erscheinungen verdeutlichen den Eindruck des Fremden. Die künstlerische Auseinandersetzung mit dem Fremden in Form von Studien, die auch für die Details der Realien in der Zeit um 1400 bereits vorausgesetzt werden dürfen, lässt den vertrauten Topos des Sarazenen immer mehr in den Hintergrund treten. ${ }^{118}$

Die Verwendung des Topos und die damit verbundenen Intentionen sind ebenso vielfältig wie die Auseinandersetzung mit den Sarazenen in der schriftlichen Überlieferung. Die Wertung scheint zwischen Bewunderung und Schrecken zu schwanken, und eine grundsätzliche Ambivalenz scheint immer mit zu schwingen. Eine Reihe von beliebig montierbaren Vorurteilen steht zur Verfügung und wird jeweils wechselnd miteinander verknüpft. Dämonische Kräfte, sexuelle Überaktivitäten, Perversionen, Bilderkulte, aber auch übergroße Weisheit, enormer Reichtum und andere Eigenschaften und Gewohnheiten werden mit dem Topos des Sarazenen ebenso verbunden, wie damit auch Juden oder andere Gruppen ausgegrenzt werden. ${ }^{119}$ Der Topos dient der Abgrenzung, ist aber - und dies wird in den Bildern mindestens unterschwellig vermittelt - nicht nur zur Schilderung des Fremden eingesetzt, sondern in ihm spiegelt sich zugleich das Eigene. Die Gleichrangigkeit der Kämpfenden in den Londoner Reiseberichten ebenso wie die Dämonisierung der Gegner in der "Histoire d'Outremer» wirft ein besonders ehrenvolles Licht auf die Kreuzritter und edelt

117 London, The British Library: Royal 19 D I, fol. 97.

118 MACK (wie Anm. 58), S. 153-170.

119 STRICKLAND (wie Anm. 14), S. 95 ff. und 157 ff. 
deren Einsatz für die gerechte Sache. Die orientalischen Potentaten oder Weisen als Berater oder Visionäre sind - sowohl in der Augustinus-Handschrift als auch im Stundenbuch des Duc de Berry - dem Codex gleichsam inkorporiert worden, um seine Wirkung als Hort des Wissens, der Belehrung und des Gebets zu stärken. Zugleich heben sie das Prestige des Besitzers, indem sie die Weiträumigkeit und Pracht seiner Sammlungstätigkeit bezeugen. Das Andere ist folglich nicht als 'Fremderfahrung' dargestellt, sondern wird im großen Vorstellungskomplex des Eigenen als topisches Muster gespeichert und kann je nach Ausrichtung des Bildprogramms unterschiedlich montiert für jeweils andere Gruppen eingesetzt werden. Am Beispiel der Bolognini-Kapelle konnte gezeigt werden, wie wenig die Mohammed-Darstellung mit dem Propheten selbst oder auch nur mit der Vorstellung der Muslime als geschlossener Gruppe zu tun hat, sondern in diesem Wandbild dazu instrumentalisiert wird, den Gegenpapst zu diskreditieren. In diesem Sinne wurde in allen untersuchten Beispielen der Topos des Sarazenen für bestimmte Zwecke eingesetzt und diente kaum je dazu, Muslime zu charakterisieren.

\section{Abbildungsnachweis:}

Abb. 1, 2, 10, 11: London, The British Library Board; Abb. 3, 4, 6, 7: Paris, Bibliothèque nationale de France; Abb. 5: Heidelberg, Renate J. Deckers-Matzko; Abb. 8, 9: (C) Faksimile Verlag/wissenmedia in der inmediaONE] GmbH Güterlsloh/München; Abb. 12: Wien, Österreichische Nationalbibliothek 